\title{
NONLINEAR SCHRÖDINGER EQUATION WITH TIME DEPENDENT POTENTIAL*
}

\author{
RÉMI CARLES ${ }^{\dagger}$
}

\begin{abstract}
We prove a global well-posedness result for defocusing nonlinear Schrödinger equations with time dependent potential. We then focus on time dependent harmonic potentials. This aspect is motivated by physics (Bose-Einstein condensation), and appears also as a preparation for the analysis of the propagation of wave packets in a nonlinear context. The main aspect considered in this paper is the growth of high Sobolev norms of the solution when the potential is exactly quadratic in space. Such a control is needed to study the large time propagation of nonlinear coherent states.
\end{abstract}

Key words. Nonlinear Schrödinger equation, time dependent external potential, Avron-Herbst formula, lens transform, large time behavior.

2010 subject classifications. 35Q55, 35A01, 35B40.

\section{Introduction}

Let $d \geqslant 1$, and for $x \in \mathbf{R}^{d}$ consider the nonlinear Schrödinger equation

$$
i \partial_{t} u+\frac{1}{2} \Delta u=V(t, x) u+\lambda|u|^{2 \sigma} u ; \quad u_{\mid t=0}=u_{0},
$$

where $V \in \mathbf{R}$ is locally bounded in time and subquadratic in space, $\lambda \in \mathbf{R}$, and the nonlinearity is energy-subcritical $(\sigma<2 /(d-2)$ if $d \geqslant 3)$. We prove that the solution exists globally and is in

$$
\Sigma=\left\{f \in H^{1}\left(\mathbf{R}^{d}\right) ; \quad x \mapsto|x| f(x) \in L^{2}\left(\mathbf{R}^{d}\right)\right\},
$$

provided that $u_{0} \in \Sigma, \sigma<2 / d$ (mass-subcritical nonlinearity), or $\sigma \geqslant 2 / d$ and $\lambda \geqslant 0$ (defocusing nonlinearity). We then focus on the case where $V$ is exactly quadratic in $x$ :

$$
i \partial_{t} u+\frac{1}{2} \Delta u=\frac{1}{2} \sum_{j=1}^{d} \Omega_{j}(t) x_{j}^{2} u+\lambda|u|^{2 \sigma} u ; \quad u_{\mid t=0}=u_{0},
$$

where $\Omega_{j} \in \mathbf{R}$, with $\Omega_{j} \in C^{1}(\mathbf{R})$. In the isotropic case $\left(\Omega_{j}=\Omega\right.$ for all $\left.j\right)$, we show that the above result is optimal in the sense that for all $\Omega \in C(\mathbf{R} ; \mathbf{R})$, if $\lambda<0$ and $\sigma=2 / d$, there exist blow-up solutions. We also investigate the growth of high Sobolev norms for large time.

There are at least two motivations to study (1.1) in general, and (1.2) in particular. In physics, this external potential may model a time dependent confining magnetic potential (which is turned on and off successively, hence the dependence in time): (1.1) and (1.2) appear in Bose-Einstein condensation, typically for $\sigma=1$ (or $\sigma=2$ sometimes in the one-dimensional case $d=1$ ); see e.g. [12, 22, 28]. Equation (1.2) also appears as an envelope equation in the nonlinear propagation of wave packets. In the linear case, consider

$$
i \varepsilon \partial_{t} \psi^{\varepsilon}+\frac{\varepsilon^{2}}{2} \Delta \psi^{\varepsilon}=V(x) \psi^{\varepsilon} ; \quad \psi^{\varepsilon}(0, x)=\frac{1}{\varepsilon^{d / 4}} \varphi\left(\frac{x-x_{0}}{\sqrt{\varepsilon}}\right) e^{i\left(x-x_{0}\right) \cdot \xi_{0} / \varepsilon} .
$$

*Received: November 21, 2010; accepted: February 8, 2011. Communicated by Peter Markowich. This work was supported by the French ANR project R.A.S. (ANR-08-JCJC-0124-01).

${ }^{\dagger}$ Univ. Montpellier 2 and CNRS, UMR 5149, Mathématiques CC 051, F-34095 Montpellier, France (Remi.Carles@math.cnrs.fr). 
In the limit $\varepsilon \rightarrow 0, \psi^{\varepsilon}$ can be approximated as follows:

$$
\psi^{\varepsilon}(t, x) \underset{\varepsilon \rightarrow 0}{\sim} \frac{1}{\varepsilon^{d / 4}} u\left(t, \frac{x-x(t)}{\sqrt{\varepsilon}}\right) e^{i \phi(t, x) / \varepsilon},
$$

where $(x(t), \xi(t))$ is given by the Hamiltonian flow associated to $H=\frac{|\xi|^{2}}{2}+V(x)$, with initial data $\left(x_{0}, \xi_{0}\right)$,

$$
\phi(t, x)=(x-x(t)) \cdot \xi(t)+\int_{0}^{t}\left(\frac{1}{2}|\xi(\tau)|^{2}-V(x(\tau))\right) d \tau,
$$

and $u$ is given by the equation

$$
i \partial_{t} u+\frac{1}{2} \Delta u=\frac{1}{2}\langle Q(t) x, x\rangle u ; \quad u_{\mid t=0}=\varphi .
$$

Here, $Q$ is defined by $Q(t)=\operatorname{Hess} V(x(t))$, the Hessian of $V$ at point $x(t)$; see e.g. [15]. We note that the external potential in this case has the form presented in (1.2). To study the nonlinear propagation of wave packets, another parameter must be taken into account: the size of the initial data. In [11], it is shown that there exists a critical size (depending on the nonlinearity and the space dimension), corresponding to a certain power of $\varepsilon$ : for initial data which are smaller (as $\varepsilon \rightarrow 0$ ) than this critical size, the nonlinearity is negligible and we retrieve the same description as above; for initial data which have the critical size, we have a similar description, up to the fact that the envelope equation is now nonlinear, of the form (1.2). To study the propagation of wave packets over large times (typically, up to an analogue of Ehrenfest time), one has to understand the large time behavior of solutions to (1.2). The main contribution of the present paper is precisely to control the growth of high order Sobolev norms, and momenta (which are related since the harmonic potential rotates the phase space, and hence exchanges space and frequency regularity; see e.g. [9]) of the solution to (1.2).

REMARK 1.1 (Time dependent nonlinearity). With little modification, we could also consider the more general equation

$$
i \partial_{t} u+\frac{1}{2} \Delta u=\frac{1}{2} \sum_{j=1}^{d} \Omega_{j}(t) x_{j}^{2} u+h(t)|u|^{2 \sigma} u ; \quad u_{\mid t=0}=u_{0},
$$

where $h \in C^{\infty}(\mathbf{R} ; \mathbf{R})$. Following [14] (see also [13]), the regularity assumption on $h$ could be weakened. We choose to consider an autonomous nonlinearity in most of this paper though.

REMARK 1.2 (Complete integrability). The cubic one-dimensional case $d=\sigma=1$ is special: if $\Omega=0$, then the equation is completely integrable ([1]). More generally, (1.3) has a Lax pair (recall that $d=\sigma=1$ ) provided that $\Omega$ and $h$ are related through the identities $([31,27])$

$$
\Omega(t)=\ddot{f}(t)-\dot{f}(t)^{2} ; \quad h(t)=a e^{f(t)} ; \quad a \in \mathbf{R}, \quad f \in C^{\infty}(\mathbf{R} ; \mathbf{R}) .
$$

The case where the above relation is not satisfied is included in Proposition 1.5.

The assumption we make on the external potential $V$ is inspired by [20]:

Assumption 1.3. $V \in L_{\mathrm{loc}}^{\infty}\left(\mathbf{R}_{t} \times \mathbf{R}_{x}^{d}\right)$ is smooth with respect to the space variable: for (almost) all $t \in \mathbf{R}, x \mapsto V(t, x)$ is a $C^{\infty}$ map. Moreover, it is subquadratic in space:

$$
\forall T>0, \forall \alpha \in \mathbf{N}^{d},|\alpha| \geqslant 2, \quad \partial_{x}^{\alpha} V \in L^{\infty}\left([-T, T] \times \mathbf{R}^{d}\right) .
$$


Note that this assumption does not involve spectral properties of $V$, and demands very little concerning the dynamical properties of the associated Hamiltonian. The time dependent harmonic potential that we consider in (1.2) is of course a peculiar case of such potentials $V$.

Before stating the main results of this paper, let us emphasize that when the potential $V$ is linear in $x$, an extension of the celebrated Avron-Herbst formula (when $V=E \cdot x$ with $E \in \mathbf{R}^{d}$ constant; see e.g. [18]) is available. The following result can be checked by straightforward computations:

Lemma 1.4 (Generalized Avron-Herbst formula). Let $E \in L_{\mathrm{loc}}^{1}\left(\mathbf{R} ; \mathbf{R}^{d}\right)$, and $h$ some function of $t$. The solutions to

$$
\begin{aligned}
& i \partial_{t} v+\frac{1}{2} \Delta v=h(t)|v|^{2 \sigma} v ; \quad v_{\mid t=0}=u_{0}, \\
& i \partial_{t} u+\frac{1}{2} \Delta u=E(t) \cdot x u+h(t)|u|^{2 \sigma} u ; \quad u_{\mid t=0}=u_{0},
\end{aligned}
$$

are related by the formula

$$
u(t, x)=v\left(t, x+\int_{0}^{t} \int_{0}^{\tau} E(s) d s d \tau\right) e^{-i x \cdot \int_{0}^{t} E(\tau) d \tau-\frac{i}{2} \int_{0}^{t}\left|\int_{0}^{s} E(\tau) d \tau\right|^{2} d s} .
$$

In particular, all the results known for (1.1) in the case $V=0$ are immediately translated to the case $V(t, x)=E(t) \cdot x$. We shall not pursue this aspect here, since our main motivation is the large time behavior of solutions to (1.2).

1.1. $L^{2}$-subcritical case. When the energy is $L^{2}$-subcritical $(\sigma<2 / d)$, we have:

Proposition 1.5. Let $\lambda \in \mathbf{R}, 0<\sigma<2 / d$, and $V$ satisfy Assumption 1.3. For all $u_{0} \in L^{2}\left(\mathbf{R}^{d}\right)$, (1.1) has a unique solution

$$
u \in C\left(\mathbf{R} ; L^{2}\left(\mathbf{R}^{d}\right)\right) \cap L_{\mathrm{loc}}^{\frac{4 \sigma+4}{d \sigma}}\left(\mathbf{R} ; L^{2 \sigma+2}\left(\mathbf{R}^{d}\right)\right) .
$$

Moreover, its $L^{2}$-norm is independent of time:

$$
\|u(t)\|_{L^{2}\left(\mathbf{R}^{d}\right)}=\left\|u_{0}\right\|_{L^{2}\left(\mathbf{R}^{d}\right)}, \quad \forall t \in \mathbf{R} .
$$

Sketch of the proof. In view of [20, 21], local in time Strichartz estimates are available. Therefore, one can reproduce the original proof of [37] (see also [13, 34]), in order to infer the result.

1.2. Energy subcritical case. In order to encompass the physical case $\sigma=1$ when $d=2$ or 3 , we need to consider the case $\sigma \geqslant 2 / d$. We shall restrict our attention to $H^{1}$-subcritical nonlinearities: $\sigma<2 /(d-2)$ when $d \geqslant 3$. To solve (1.2), even locally in time, one needs to work in $\Sigma$, and not only $H^{1}$; symmetry is needed on physical and frequency sides, unless $V$ is sublinear [9]. Local existence in $\Sigma$ then follows from the dispersive estimates in [20,21]; one can work as in the case $V \equiv 0$ (where it is possible to work in $H^{1}\left(\mathbf{R}^{d}\right)$ only). Instead of considering only $(u, \nabla u)$ as the unknown function, one can consider $(u, \nabla u, x u)$. The three functions are related through a closed family of estimates, and we get: 
Proposition 1.6. Let $\lambda \in \mathbf{R}, V$ be a potential satisfying Assumption 1.3, and $\sigma>0$, with $\sigma<2 /(d-2)$ if $d \geqslant 3$. For $u_{0} \in \Sigma$, there exists $T$ and a unique solution $u$ to (1.1) such that

$$
u, \nabla u, x u \in C(]-T, T\left[; L^{2}\left(\mathbf{R}^{d}\right)\right) \cap L_{\mathrm{loc}}^{\frac{4 \sigma+4}{d \sigma}}(]-T, T\left[; L^{2 \sigma+2}\left(\mathbf{R}^{d}\right)\right) .
$$

Moreover, its $L^{2}$-norm is independent of time:

$$
\left.\|u(t)\|_{L^{2}\left(\mathbf{R}^{d}\right)}=\left\|u_{0}\right\|_{L^{2}\left(\mathbf{R}^{d}\right)}, \quad \forall t \in\right]-T, T[.
$$

Since only bounded time intervals are considered in [20,21], we give a more precise treatment of this result in $\S 2$ in order to consider global in time solutions. We have been careful in the statement of Proposition 1.6 not to write that $T$ depends only on $\left\|u_{0}\right\|_{\Sigma}$. To infer global existence results, we wish to replace the initial time $t=0$ in (1.1) with $t=t_{0} \geqslant 0$. Under the assumptions of Proposition 1.6, it is not guaranteed that the corresponding $T$ is independent of $t_{0}$. However, it is proved in $[20,21]$ that local dispersive estimates are available, uniformly on finite time intervals.

The natural candidate for an energy in the case of (1.1) is

$$
E(t)=\frac{1}{2}\|\nabla u(t)\|_{L^{2}}^{2}+\frac{\lambda}{\sigma+1}\|u(t)\|_{L^{2 \sigma+2}}^{2 \sigma+2}+\int_{\mathbf{R}^{d}} V(t, x)|u(t, x)|^{2} d x .
$$

Proposition 1.7. Under the assumptions of Proposition 1.6, if in addition $V$ is $C^{1}$ with respect to $t$, and $\partial_{t} V$ satisfies Assumption 1.3, then $E \in C^{1}(]-T, T[; \mathbf{R})$, and its evolution is given by

$$
\frac{d E}{d t}=\int_{\mathbf{R}^{d}} \partial_{t} V(t, x)|u(t, x)|^{2} d x .
$$

The proof of the above result is straightforward, and follows the same lines as in the justification of similar evolution laws in, e.g., [13].

TheOREm 1.8 (Global existence in $\Sigma$ ). Let $\lambda \in \mathbf{R}, \sigma>0$ with $\sigma<2 /(d-2)$ if $d \geqslant 3$, and let $V$ satisfy Assumption 1.3. For $u_{0} \in \Sigma$, we can take $T=+\infty$ in Proposition 1.6 in the following cases:

- $\sigma<2 / d$.

- $\sigma \geqslant 2 / d$ and $\lambda \geqslant 0$, provided $V$ is $C^{1}$ in $t$ and $\partial_{t} V$ satisfies Assumption 1.3.

REMARK 1.9. This result extends the main one in [7], where typically the (time independent) potential $-\omega_{1}^{2} x_{1}^{2}+\omega_{2}^{2} x_{2}^{2}$ is considered. It is established in [7] that if $\lambda>0$ and $\omega_{1} \gg 1+\omega_{2}$, then the solution to (1.2) is global, and there is scattering. The present theorem extends the existence part, but of course the assumptions of Theorem 1.8 are too general to expect a scattering result; in the case of (1.2) with $\Omega_{j}=1 \forall j$, for instance, one can construct periodic solutions to (1.2), of the form $u(t, x)=e^{-i \omega t} \psi(x)$. Indeed, this amounts to finding a non-trivial solution to the elliptic problem

$$
\omega \psi=H \psi+\lambda|\psi|^{2 \sigma} \psi, \text { where } H=-\frac{1}{2} \Delta+\frac{|x|^{2}}{2} .
$$

Introduce the quantities

$$
\begin{aligned}
I(\psi) & =\frac{1}{2}\langle H \psi, \psi\rangle-\frac{\omega}{2}\langle\psi, \psi\rangle, \\
M & =\left\{\psi \in \Sigma ; \frac{1}{\sigma+1} \int_{\mathbf{R}^{d}}|\psi(x)|^{2 \sigma+2} d x=1\right\},
\end{aligned}
$$


and consider

$$
\delta=\inf _{\psi \in M} I(\psi)
$$

If $\omega>d / 2$ (the lowest eigenvalue of the harmonic oscillator), then (1.6) has a nontrivial solution for $\lambda>0$. If $\omega<d / 2$, then (1.6) has a non-trivial solution for $\lambda<0$. See e.g. [10] for more details.

Theorem 1.8 shows that in the usual cases where global existence is known without a potential, the introduction of a smooth subquadratic potential $V$ does not change this property, regardless of the time dependence of $V$. In the case $\sigma=2 / d$ and $\lambda<0$, we prove that finite time blow-up does occur for time dependent potentials, like in the case with no potential:

Proposition 1.10 (Finite time blow-up). Let $\sigma=2 / d$ and $\lambda<0$. Consider (1.2) with an isotropic potential: $\Omega_{j}=\Omega \in C(\mathbf{R} ; \mathbf{R})$ is independent of $j$. There exist blow-up solutions for (1.2). That is, we can find $T>0$, and $u \in C(10, T] ; \Sigma)$ solving (1.2) such that

$$
\|\nabla u(t)\|_{L^{2}} \underset{t \rightarrow 0}{\longrightarrow} \infty
$$

1.3. Growth of higher order Sobolev norms. We now focus on the case of time dependent harmonic potentials, (1.2). In view of the analysis of nonlinear wave packets in a semi-classical regime ([11]), the evolution of weighted Sobolev norms of $u$ over large time intervals is needed.

Consider first the autonomous isotropic case: $\Omega_{j}=\Omega$ is a constant.

If $\Omega=0$, then when $\lambda \geqslant 0$ and $2 / d \leqslant \sigma<2 /(d-2)$ the Sobolev norms of $u$ are bounded, $u \in L^{\infty}\left(\mathbf{R} ; H^{k}\left(\mathbf{R}^{d}\right)\right)$ (provided the nonlinearity is sufficiently smooth), since we know that there is scattering in $H^{1}$ (because we know that there is scattering in $\Sigma$, since scattering in $H^{1}$ only is not known so far in the case $\sigma=2 / d$ ); see e.g. [38]. The momenta of $u$ grow algebraically in time (see [2]). We give a short alternative proof of these properties in an appendix.

If $\Omega>0$, then $u \in L^{\infty}(\mathbf{R} ; \Sigma)$, as proved by (1.5). The existence of periodic solutions to the nonlinear problem (see Remark 1.9) shows that we may also have $u \in L^{\infty}\left(\mathbf{R} ; H^{k}\right)$ and $|x|^{k} u \in L^{\infty}\left(\mathbf{R} ; L^{2}\right)$ for all $k \in \mathbf{N}$.

If $\Omega<0$ (repulsive harmonic potential), then it is proved in [6] that every defocusing $H^{1}$-subcritical nonlinearity is short range as far as scattering theory is concerned; if $\lambda>0$ in (1.2), then $u(t) \sim U(t) u_{+}$as $t \rightarrow+\infty$, for some $u_{+} \in \Sigma$, where $U(t)=\exp \left(-i t\left(-\frac{1}{2} \Delta+\frac{\Omega}{2}|x|^{2}\right)\right)$. Assume $\Omega=-1$. Using Mehler's formula, and a decomposition of $U(t)$ of the form $U=M D \mathcal{F} M$ as in [36] (for the case $\Omega=0$ ), we notice

$$
U(t) u_{+}(x) \underset{t \rightarrow+\infty}{\sim} \frac{1}{\sinh t} \mathcal{F}\left(u_{+} e^{|\cdot|^{2} / 2}\right)\left(\frac{x}{\sinh t}\right) e^{i \frac{\cosh t}{\sinh t} \frac{|x|^{2}}{2}} .
$$

This shows that the $L^{2}$ norms of $\nabla U(t) u_{+}$and $x U(t) u_{+}$grow exponentially in time. By the results in [6], so do the $L^{2}$ norms of $\nabla u$ and $x u$. Note that at least in the linear case $\lambda=0$, we see that the $H^{k}$-norms of $u$ grow like $e^{k t}$ as $t$ goes to infinity.

Definition 1.11. Let $u \in C(\mathbf{R} ; \Sigma)$ be a solution to $(1.2)$, and $k \in \mathbf{N}$.

- $(A l g)_{k}$ is satisfied if there exists $A$ such that for all admissible pair $(p, q)$,

$$
\forall \alpha, \beta \in \mathbf{N}^{d},|\alpha|+|\beta| \leqslant k, \quad\left\|x^{\alpha} \partial_{x}^{\beta} u\right\|_{L^{p}\left([0, t] ; L^{q}\right)} \lesssim t^{A} .
$$


- $(E x p)_{k}$ is satisfied if there exists $C$ such that for all admissible pair $(p, q)$,

$$
\forall \alpha, \beta \in \mathbf{N}^{d},|\alpha|+|\beta| \leqslant k, \quad\left\|x^{\alpha} \partial_{x}^{\beta} u\right\|_{L^{p}\left([0, t] ; L^{q}\right)} \lesssim e^{C t} .
$$

We wish to consider smooth energy-subcritical nonlinearities. Since we study homogeneous nonlinearities, we have to assume: $d \leqslant 3, \sigma \in \mathbf{N}$, with $\sigma=1$ if $d=3$.

Corollary 1.12. Let $d \leqslant 3, \lambda \geqslant 0$ and $\sigma \in \mathbf{N}$, with $\sigma=1$ if $d=3$. Let $k \in \mathbf{N}$,

$$
u_{0} \in H^{k}\left(\mathbf{R}^{d}\right) \text {, with }|x|^{k} u_{0} \in L^{2}\left(\mathbf{R}^{d}\right) .
$$

If $\Omega_{j} \in C^{1}(\mathbf{R} ; \mathbf{R})$ is compactly supported for all $j$, then u has the property $(A l g)_{k}$.

Proof. We may assume that $\operatorname{supp} \Omega \subset[-M, M]$. By Theorem 1.8, $u_{\mid t=M} \in \Sigma$. It is easy to check that the higher regularity is conserved as well. This is straightforward, since we consider an energy-subcritical nonlinearity. The corollary then follows from the case $\Omega=0$, where $(A l g)_{k}$ is satisfied, as recalled in the appendix.

In the case where the dependence of $\Omega_{j}$ with respect to time is not specified, the evolution of the energy (1.4) yields no exploitable information. Even in the $L^{2}$ subcritical case, we will see that proving exponential control requires some work.

Proposition 1.13 (Exponential growth). Let $d \leqslant 3, \sigma \in \mathbf{N}$, with $\sigma=1$ if $d=3$, $\Omega_{j} \in C(\mathbf{R} ; \mathbf{R})$ be locally Lipschitz, $k \in \mathbf{N}, k \geqslant 1$, and

$$
u_{0} \in H^{k}\left(\mathbf{R}^{d}\right), \text { with }|x|^{k} u_{0} \in L^{2}\left(\mathbf{R}^{d}\right) .
$$

$(\text { Exp })_{k}$ is satisfied (at least) in the following cases:

- $\sigma=d=1$ ( $L^{2}$-subcritical nonlinearity), and $\Omega$ is bounded.

- $\sigma \geqslant 2 / d, \lambda \geqslant 0$, and $\Omega_{j}=\Omega \leqslant 0$ is independent of $j$ (isotropic repulsive potential).

REMARK 1.14 (Optimality). When the potential is repulsive and timeindependent ( $\Omega=-1$ typically), the exponential growth is sharp, and $C$ does depend on $k(C=k$ when $\Omega=-1)$, as discussed above.

REMARK 1.15. We prove that in the case of an isotropic repulsive potential, there is scattering provided $\sigma \geqslant 2 / d$ (Proposition 6.4): morally, $(\operatorname{Exp})_{k}$ is satisfied because it is satisfied in the linear setting (case $\lambda=0$ ). However, this property on the linear solution demands a justification; the key is Lemma 6.2.

1.4. Outline of the paper. In Section 2, we prove Theorem 1.8. We then focus on the study of Equation (1.2). In Section 3, we derive a generalized Mehler formula to express the fundamental solution associated to the linear equation (1.2) with $\lambda=0$. In Section 4 , we generalize a lens transform, known in the case of isotropic timeindependent quadratic potentials, to the case of isotropic time-dependent quadratic potentials. This allows us to infer Proposition 1.10. In Section 5, we introduce some vector fields, corresponding to Heisenberg derivatives, which yield interesting evolution laws when the potential is isotropic. In Section 6, we examine the large time behavior of solutions to (1.2), and prove Proposition 1.13. Finally, we show in an appendix that when $V=0$, for large time, the solutions to (1.1) have bounded Sobolev norms and algebraically growing momenta, provided there is scattering. 


\section{Global existence in $\Sigma$ : proof of Theorem 1.8}

2.1. Strichartz estimates. We first recall some results established in [20,21]. Consider $V$ satisfying Assumption 1.3. It is established in [20] that one can define $U(t, s)$ as $u(t, x)=U(t, s) \varphi(x)$, where

$$
i \partial_{t} u+\frac{1}{2} \Delta u=V(t, x) u ; \quad u(s, x)=\varphi(x),
$$

along with the following properties:

- $U(t, t)=\mathrm{Id}$.

- The map $(t, s) \mapsto U(t, s)$ is strongly continuous.

- $U(t, s)^{*}=U(t, s)^{-1}$.

- $U(t, \tau) U(\tau, s)=U(t, s)$.

- $U(t, s)$ is unitary on $L^{2}:\|U(t, s) \varphi\|_{L^{2}}=\|\varphi\|_{L^{2}}$.

In addition, we know from [21] that for all $T>0, t, s \in[-T, T]$,

$$
\left\|U(t, 0) U(s, 0)^{*} \varphi\right\|_{L^{\infty}\left(\mathbf{R}^{d}\right)}=\|U(t, s) \varphi\|_{L^{\infty}\left(\mathbf{R}^{d}\right)} \leqslant \frac{C}{|t-s|^{d / 2}}\|\varphi\|_{L^{1}\left(\mathbf{R}^{d}\right)},
$$

provided that $|t-s|<\eta$. It is implicitly assumed in [20] that $\eta$ may depend on $T$; in Example 2.4 below, we show that indeed it does in the case of the time dependent harmonic potential if the functions $\Omega_{j}$ are not bounded.

Recall the standard definition in the context of Schrödinger equations:

Definition 2.1. A pair $(p, q)$ is admissible if $2 \leqslant q<\frac{2 d}{d-2}(2 \leqslant q \leqslant \infty$ if $d=1,2 \leqslant q<\infty$ if $d=2$ ) and

$$
\frac{2}{p}=\delta(q):=d\left(\frac{1}{2}-\frac{1}{q}\right)
$$

The general results on Strichartz estimates (see e.g. [26]) then yield, as a consequence of the dispersive estimate (2.2):

Proposition 2.2. Recall that $U(t, s)$ is defined by (2.1), where $V$ satisfies Assumption 1.3. Let $T>0$. There exists $\eta>0$ such that the following holds:

(1) For any admissible pair $(p, q)$, there exists $C_{q}$ such that

$$
\left.\|U(\cdot, s) \varphi\|_{L^{p}\left([s, s+\eta] ; L^{q}\right)} \leqslant C_{q}\|\varphi\|_{L^{2}}, \quad \forall \varphi \in L^{2}\left(\mathbf{R}^{d}\right), \quad \forall s \in\right]-T, T-\eta[.
$$

(2) For $s \in \mathbf{R}$, denote

$$
D_{s}(F)(t, x)=\int_{s}^{t} U(t, \tau) F(\tau, x) d \tau
$$

For all admissible pairs $\left(p_{1}, q_{1}\right)$ and $\left(p_{2}, q_{2}\right)$, there exists $C=C_{q_{1}, q_{2}}$ independent of $s \in]-T, T-\eta[$ such that

$$
\left\|D_{s}(F)\right\|_{L^{p_{1}\left([s, s+\delta] ; L^{q_{1}}\right)}} \leqslant C\|F\|_{L^{p_{2}^{\prime}}\left([s, s+\delta] ; L^{q_{2}^{\prime}}\right)},
$$

for all $F \in L^{p_{2}^{\prime}}\left(I ; L^{q_{2}^{\prime}}\right)$ and $0 \leqslant \delta \leqslant \eta$. 
EXAMPLE 2.3 (Standard harmonic oscillator). Assume that $V(t, x)=\frac{|x|^{2}}{2}$. The above result is then standard (see e.g. [13]). The fact that one has to consider finite time intervals for the above result to be valid stems for instance from the existence of eigenvalues for the harmonic oscillator: let $g(x)=e^{-|x|^{2} / 2}$ be the ground state associated to the harmonic potential, and denote $u(t, x)=e^{-i t d / 2} g(x)$. It solves

$$
i \partial_{t} u+\frac{1}{2} \Delta u=\frac{|x|^{2}}{2} u ; \quad u_{\mid t=0}=g .
$$

We compute $\|u\|_{L^{p}\left(I ; L^{q}\right)}=|I|^{1 / p}\|g\|_{L^{q}}$, which shows that Proposition 2.2 becomes false with $\eta=\infty$.

EXAMPLE 2.4. We show that in general, the above result is false with $T=\infty$. Let

$$
V(t, x)=\frac{1}{2} \Omega(t)|x|^{2} .
$$

If $\Omega$ is not bounded, then the above uniformity with respect to $s$ fails: let

$$
\Omega(t)=n^{2} \text { if } 4 n+1=t_{n} \leqslant t \leqslant 4 n+2 .
$$

Since we have

$$
\left(-\frac{1}{2} \Delta+\frac{n^{2}}{2}|x|^{2}\right) e^{-n|x|^{2} / 2}=\frac{n d}{2} e^{-n|x|^{2} / 2}
$$

the function $u(t, x)=e^{-i n d\left(t-t_{n}\right) / 2-n|x|^{2} / 2}$ solves (2.1) with $s=t_{n}$. If Proposition 2.2 was true with $T=\infty$, we would have:

$$
\|u\|_{L^{p}\left([4 n+1,4 n+1+\eta] ; L^{q}\right)}=\eta^{1 / p}\left(\frac{2 \pi}{n q}\right)^{d /(2 q)} \leqslant C\left\|u\left(t_{n}\right)\right\|_{L^{2}}=C\left(\frac{\pi}{n}\right)^{d / 4},
$$

where $C$ does not depend on $n$. For all $q>2$, letting $n$ go to infinity leads to a contradiction. Since (2.2) implies Proposition 2.2, this shows that (2.2) is valid for $|t-s|<\eta$, where $\eta$ depends on $T$, unless $\Omega$ is bounded.

2.2. Local existence in $\Sigma$. Since (1.1) is not autonomous, we consider the same problem with a varying initial time:

$$
i \partial_{t} u+\frac{1}{2} \Delta u=V(t, x) u+\lambda|u|^{2 \sigma} u ; \quad u_{\mid t=s}=u_{0},
$$

with $s \in \mathbf{R}$.

Proposition 2.5. Let $\lambda \in \mathbf{R}, \sigma>0$ with $\sigma<2 /(d-2)$ if $d \geqslant 3$, and let $V$ satisfy Assumption 1.3. Let $M>0$, and $s \in]-M, M\left[\right.$. For all $u_{0} \in \Sigma$, there exists $T=T\left(\left\|u_{0}\right\|_{\Sigma}, M\right)$ and a unique solution $u$ solution to (2.4), such that

$$
u, \nabla u, x u \in C(] s-T, s+T\left[; L^{2}\left(\mathbf{R}^{d}\right)\right) \cap L_{\mathrm{loc}}^{\frac{4 \sigma+4}{d \sigma}}(] s-T, s+T\left[; L^{2 \sigma+2}\left(\mathbf{R}^{d}\right)\right) .
$$

Moreover, its $L^{2}$-norm is independent of time:

$$
\left.\|u(t)\|_{L^{2}\left(\mathbf{R}^{d}\right)}=\left\|u_{0}\right\|_{L^{2}\left(\mathbf{R}^{d}\right)}, \quad \forall t \in\right] s-T, s+T[.
$$

If $V$ is $C^{1}$ in $t$, then the energy $E$ (defined by (1.4)) evolves according to (1.5). 
Sketch of the proof. We present here only the main steps of the classical argument. Duhamel's formulation for (2.4) reads

$$
u(t)=U(t, s) u_{0}-i \lambda \int_{s}^{t} U(t, \tau)\left(|u|^{2 \sigma} u\right)(\tau) d \tau
$$

Denote the right hand side by $\Phi^{s}(u)(t)$. Proposition 2.5 follows from a fixed point argument in the space

$$
X_{T}=\left\{u \in C\left(I_{T} ; \Sigma\right) ; u, x u, \nabla u \in L^{\frac{4 \sigma+4}{d \sigma}}\left(I_{T} ; L^{2 \sigma+2}\left(\mathbf{R}^{d}\right)\right)\right\},
$$

where $\left.I_{T}=\right] s-T, s+T[$. Introduce the Lebesgue exponents

$$
q=2 \sigma+2 ; \quad p=\frac{4 \sigma+4}{d \sigma} ; \quad \theta=\frac{2 \sigma(2 \sigma+2)}{2-(d-2) \sigma} .
$$

Then $(p, q)$ is admissible, and

$$
\frac{1}{q^{\prime}}=\frac{2 \sigma}{q}+\frac{1}{q} ; \quad \frac{1}{p^{\prime}}=\frac{2 \sigma}{\theta}+\frac{1}{p} .
$$

Proposition 2.2 and Hölder inequality yield

$$
\begin{aligned}
\left\|\Phi^{S}(u)\right\|_{L^{p}\left(I_{T} ; L^{q}\right) \cap L^{\infty}\left(I_{T} ; L^{2}\right)} & \leqslant C\left\|u_{0}\right\|_{L^{2}}+C\left\||u|^{2 \sigma} u\right\|_{L^{p^{\prime}}\left(I_{T} ; L^{q^{\prime}}\right)} \\
& \leqslant C\left\|u_{0}\right\|_{L^{2}}+C\|u\|_{L^{\theta}\left(I_{T} ; L^{q}\right)}^{2 \sigma}\|u\|_{L^{p}\left(I_{T} ; L^{q}\right)}
\end{aligned}
$$

where $C$ is independent of $s \in[-M, M]$ and $T \leqslant \eta$. Using Sobolev embedding,

$$
\left\|\Phi^{s}(u)\right\|_{L^{p}\left(I_{T} ; L^{q}\right) \cap L^{\infty}\left(I_{T} ; L^{2}\right)} \leqslant C\left\|u_{0}\right\|_{L^{2}}+C T^{2 \sigma / \theta}\|u\|_{L^{\infty}\left(I_{T} ; H^{1}\right)}^{2 \sigma}\|u\|_{L^{p}\left(I_{T} ; L^{q}\right)} .
$$

We have

$$
\begin{aligned}
\nabla \Phi^{s}(u)(t)= & U(t, s) \nabla u_{0}-i \lambda \int_{s}^{t} U(t, \tau) \nabla\left(|u|^{2 \sigma} u\right)(\tau) d \tau \\
& -i \int_{s}^{t} U(t, \tau)\left(\Phi^{s}(u)(\tau) \nabla V(\tau)\right) d \tau
\end{aligned}
$$

We estimate the second term of the right hand side as above and get, since $\nabla V$ is sublinear by assumption,

$$
\begin{aligned}
\left\|\nabla \Phi^{s}(u)\right\|_{L^{p}\left(I_{T} ; L^{q}\right) \cap L^{\infty}\left(I_{T} ; L^{2}\right)} \leqslant & C\left\|\nabla u_{0}\right\|_{L^{2}}+C T^{2 \sigma / \theta}\|u\|_{L^{\infty}\left(I_{T} ; H^{1}\right)}^{2 \sigma}\|\nabla u\|_{L^{p}\left(I_{T} ; L^{q}\right)} \\
& +C\left\|\Phi^{s}(u) \nabla V\right\|_{L^{1}\left(I_{T} ; L^{2}\right)} \\
\leqslant & C\left\|\nabla u_{0}\right\|_{L^{2}}+C T^{2 \sigma / \theta}\|u\|_{L^{\infty}\left(I_{T} ; H^{1}\right)}^{2 \sigma}\|\nabla u\|_{L^{p}\left(I_{T} ; L^{q}\right)} \\
& +C T\left\|x \Phi^{s}(u)\right\|_{L^{\infty}\left(I_{T} ; L^{2}\right)}+C T\left\|\Phi^{s}(u)\right\|_{L^{\infty}\left(I_{T} ; L^{2}\right)}
\end{aligned}
$$

where, again, $C$ does not depend on $s \in[-M, M]$. We have similarly

$$
\begin{aligned}
\left\|x \Phi^{s}(u)\right\|_{L^{p}\left(I_{T} ; L^{q}\right) \cap L^{\infty}\left(I_{T} ; L^{2}\right)} \leqslant & C\left\|x u_{0}\right\|_{L^{2}}+C T^{2 \sigma / \theta}\|u\|_{L^{\infty}\left(I_{T} ; H^{1}\right)}^{2 \sigma}\|x u\|_{L^{p}\left(I_{T} ; L^{q}\right)} \\
& +C T\left\|\nabla \Phi^{s}(u)\right\|_{L^{\infty}\left(I_{T} ; L^{2}\right)} .
\end{aligned}
$$


Choosing $T$ sufficiently small, one can then prove that $\Phi^{s}$ maps a suitable ball in $X_{T}$ into itself. Contraction for the norm $\|\cdot\|_{L^{p}\left(I_{T} ; L^{q}\right)}$ is proved similarly, and one concludes by remarking that $X_{T}$ equipped with this norm is complete.

We can now infer the analogue to the standard result (which is not straightforward since we consider a non-autonomous equation, in the presence of an external potential):

Corollary 2.6. Let $\lambda \in \mathbf{R}, \sigma>0$ with $\sigma<2 /(d-2)$ if $d \geqslant 3$, V satisfy Assumption 1.3, and $u_{0} \in \Sigma$. Either the solution to (1.2) is global in time (in the future),

$$
u, \nabla u, x u \in C\left(\mathbf{R}_{+} ; L^{2}\left(\mathbf{R}^{d}\right)\right) \cap L_{\mathrm{loc}}^{\frac{4 \sigma+4}{\sigma \sigma}}\left(\mathbf{R}_{+} ; L^{2 \sigma+2}\left(\mathbf{R}^{d}\right)\right),
$$

or there exists $T>0$, such that

$$
\|\nabla u(t)\|_{L^{2} \underset{t \rightarrow T}{\longrightarrow}+\infty}+\infty
$$

Proof. Let $M>0$. Proposition 2.5 shows that the only obstruction to wellposedness on $[0, M]$ is the existence of a time $0<T<M$ such that

$$
\|x u(t)\|_{L^{2}}+\|\nabla u(t)\|_{L^{2}} \underset{t \rightarrow T}{\longrightarrow}+\infty .
$$

So long as $u \in C([0, t] ; \Sigma)$, we have (see e.g. [13] for the arguments that make the computation rigorous)

$$
\frac{d}{d t} \int_{\mathbf{R}^{d}} x_{j}^{2}|u(t, x)|^{2} d x=2 \operatorname{Im} \int_{\mathbf{R}^{d}} x_{j} \bar{u}(t, x) \partial_{j} u(t, x) d x .
$$

Suppose $u \in L^{\infty}\left([0, T] ; H^{1}\right)$. Then the above formula, Cauchy-Schwarz inequality, and Gronwall's Lemma show that $x u \in L^{\infty}\left([0, T] ; L^{2}\right)$, a contradiction. The corollary follows since $M>0$ is arbitrary.

Therefore, to prove global existence in $\Sigma$ in the $H^{1}$-subcritical case, it suffices to exhibit a priori bounds for $\nabla u$ in $L^{2}$.

2.3. $L^{2}$-subcritical case. In the case $\sigma<2 / d$, recall that the classical argument of [37] can be applied directly to infer Proposition 1.5. The a priori bound for $(\nabla u, x u)$ in $L^{2}$ then follows by resuming the computations presented in the proof of Proposition 2.5. Keeping the same notations, we have in particular

$$
\begin{aligned}
& \left\|\nabla \Phi^{s}(u)\right\|_{L^{p}\left(I_{T} ; L^{q}\right) \cap L^{\infty}\left(I_{T} ; L^{2}\right)}+\left\|x \Phi^{s}(u)\right\|_{L^{p}\left(I_{T} ; L^{q}\right) \cap L^{\infty}\left(I_{T} ; L^{2}\right)} \\
& \leqslant C\left\|u_{0}\right\|_{\Sigma}+C\|u\|_{L^{\theta}\left(I_{T} ; L^{q}\right)}^{2 \sigma}\left(\|\nabla u\|_{L^{p}\left(I_{T} ; L^{q}\right)}+\|x u\|_{L^{p}\left(I_{T} ; L^{q}\right)}\right) \\
& \quad+C T\left(\left\|\Phi^{s}(u)\right\|_{L^{\infty}\left(I_{T} ; L^{2}\right)}+\left\|x \Phi^{s}(u)\right\|_{L^{\infty}\left(I_{T} ; L^{2}\right)}+\left\|\nabla \Phi^{s}(u)\right\|_{L^{\infty}\left(I_{T} ; L^{2}\right)}\right),
\end{aligned}
$$

where we recall that

$$
q=2 \sigma+2 ; \quad p=\frac{4 \sigma+4}{d \sigma} ; \quad \theta=\frac{2 \sigma(2 \sigma+2)}{2-(d-2) \sigma},
$$

and, in view of Proposition 2.5, we know that $u=\Phi^{s}(u)$. In the case $\sigma<2 / d$, we have $1 / p<1 / \theta$, and thus

$$
\|u\|_{L^{\theta}\left(I_{T} ; L^{q}\right)} \leqslant(2 T)^{1 / \theta-1 / p}\|u\|_{L^{p}\left(I_{T} ; L^{q}\right)}=(2 T)^{\frac{(2-d \sigma)(\sigma+1)}{2 \sigma(2 \sigma+2)}}\|u\|_{L^{p}\left(I_{T} ; L^{q}\right)} .
$$


By Proposition 1.5, $u \in L_{\text {loc }}^{p}\left(\mathbf{R} ; L^{q}\left(\mathbf{R}^{d}\right)\right)$. Splitting any given time interval $[-M, M]$ into finitely many (tiny) pieces, we obtain an a priori bound for $(\nabla u, x u)$ in $L^{\infty}\left([-M, M] ; L^{2}\right)$. Since $M>0$ is arbitrary, Corollary 2.6 yields the first point of Theorem 1.8.

2.4. Defocusing energy-subcritical case. We now consider the case $\lambda \geqslant 0$, with $\sigma<2 /(d-2)$ if $d \geqslant 3$. To complete the proof of Theorem 1.8, we resume the computation initiated in the proof of Corollary 2.6 in order to infer a virial identity:

Lemma 2.7. Let $\lambda \in \mathbf{R}, \sigma>0$ with $\sigma<2 /(d-2)$ if $d \geqslant 3$, and $V$ satisfy Assumption 1.3. Let $u_{0} \in \Sigma$, and $u \in C(]-T, T[; \Sigma)$ be the solution to (1.1) given by Proposition 2.5 (case $s=0)$. Denote

$$
y(t)=\int_{\mathbf{R}^{d}}|x|^{2}|u(t, x)|^{2} d x .
$$

Then $y \in C^{2}(]-T, T[)$, and satisfies

$$
\frac{d^{2} y}{d t^{2}}=2\|\nabla u(t)\|_{L^{2}}^{2}-2 \int_{\mathbf{R}^{d}} x \cdot \nabla V(t, x)|u(t, x)|^{2} d x+2 \lambda \frac{d \sigma}{\sigma+1}\|u(t)\|_{L^{2 \sigma+2}}^{2 \sigma+2} .
$$

Proof. We present the formal part of the proof, and refer to [13] for the arguments that make the proof rigorous. We first resume the computation made in the course of the proof of Corollary 2.6. Differentiating (2.5) again with respect to time, we have:

$$
\begin{aligned}
\frac{d^{2}}{d t^{2}}\left\|x_{j} u\right\|_{L^{2}}^{2} & =2 \operatorname{Im} \int_{\mathbf{R}^{d}} x_{j} \partial_{t} \bar{u} \partial_{j} u+2 \operatorname{Im} \int_{\mathbf{R}^{d}} x_{j} \bar{u} \partial_{j} \partial_{t} u \\
& =-2 \operatorname{Im} \int_{\mathbf{R}^{d}}\left(\bar{u}+2 x_{j} \partial_{j} \bar{u}\right) \partial_{t} u=2 \operatorname{Re} \int_{\mathbf{R}^{d}}\left(\bar{u}+2 x_{j} \partial_{j} \bar{u}\right) i \partial_{t} u \\
& =2 \operatorname{Re} \int_{\mathbf{R}^{d}}\left(\bar{u}+2 x_{j} \partial_{j} \bar{u}\right)\left(-\frac{1}{2} \Delta u+V(t, x) u+\lambda|u|^{2 \sigma} u\right)
\end{aligned}
$$

The terms in the right hand side simplify easily, and we infer:

$$
\begin{gathered}
\frac{d^{2}}{d t^{2}}\left\|x_{j} u\right\|_{L^{2}}^{2}=\|\nabla u\|_{L^{2}}^{2}+2 \int_{\mathbf{R}^{d}} V(t, x)|u(t, x)|^{2} d x+2 \lambda\|u\|_{L^{2 \sigma+2}}^{2 \sigma+2} \\
\quad-2 \operatorname{Re} \int_{\mathbf{R}^{d}} x_{j} \partial_{j} \bar{u} \Delta u+4 \operatorname{Re} \int_{\mathbf{R}^{d}} V(t, x) x_{j} u \partial_{j} \bar{u} \\
\quad+4 \lambda \operatorname{Re} \int_{\mathbf{R}^{d}} x_{j}|u|^{2 \sigma} u \partial_{j} \bar{u} \\
=\|\nabla u\|_{L^{2}}^{2}+2 \int_{\mathbf{R}^{d}} V(t, x)|u(t, x)|^{2} d x+2 \lambda\|u\|_{L^{2 \sigma+2}}^{2 \sigma+2} \\
\quad-\|\nabla u\|_{L^{2}}^{2}+2\left\|\partial_{j} u\right\|_{L^{2}}^{2}+2 \int_{\mathbf{R}^{d}} x_{j} V(t, x) \partial_{j}\left(|u|^{2}\right) \\
\quad-\frac{2 \lambda}{\sigma+1}\|u\|_{L^{2 \sigma+2}}^{2 \sigma+2} \\
=2\left\|\partial_{j} u\right\|_{L^{2}}^{2}-2 \int_{\mathbf{R}^{d}} x_{j} \partial_{j} V(t, x)|u(t, x)|^{2} d x+2 \lambda \frac{\sigma}{\sigma+1}\|u\|_{L^{2 \sigma+2}}^{2 \sigma+2} .
\end{gathered}
$$

The result then follows by summing over $j$. 
To complete the proof of Theorem 1.8, fix $M>0$, and for $t \in[0, M]$, let

$$
f(t)=y(t)+|\dot{y}(t)|
$$

We have

$$
\dot{f}(t) \leqslant|\dot{y}(t)|+|\ddot{y}(t)| \leqslant|\dot{y}(t)|+2\|\nabla u\|_{L^{2}}^{2}+C+C y(t)+C\|u\|_{L^{2 \sigma+2}}^{2 \sigma+2},
$$

where we have used Lemma 2.7, the estimate

$$
|x \cdot \nabla V(t, x)| \leqslant C(M)\left(1+|x|^{2}\right), \quad \forall(t, x) \in[0, M] \times \mathbf{R}^{d},
$$

and the conservation of mass. Since $u_{0} \in \Sigma,(1.4)-(1.5)$ (this is where we have to assume that $V$ is $C^{1}$ in $t$ ) yield

$$
\|\nabla u\|_{L^{2}}^{2}+\|u\|_{L^{2 \sigma+2}}^{2 \sigma+2} \lesssim 1+y(t)+\sup _{0 \leqslant s \leqslant t} y(s) \lesssim 1+\sup _{0 \leqslant s \leqslant t} y(s) .
$$

Gronwall's Lemma implies $f \in L^{\infty}([0, M])$. We infer $y \in L_{\text {loc }}^{\infty}(\mathbf{R})$. With the above inequality, this implies $\nabla u \in L_{\mathrm{loc}}^{\infty}\left(\mathbf{R} ; L^{2}\right)$, and Theorem 1.8 then follows from Corollary 2.6.

\section{Generalized Mehler formula}

In the rest of this paper, we consider the case where $V$ is exactly quadratic in $x$, and study some properties associated to (1.2).

3.1. The formula. Classically, Mehler's formula refers to the explicit formula for the fundamental solution of the linear equation

$$
i \partial_{t} u_{\operatorname{lin}}+\frac{1}{2} \Delta u_{\operatorname{lin}}=\frac{1}{2} \sum_{j=1}^{d} \Omega_{j}(t) x_{j}^{2} u_{\operatorname{lin}} ; \quad u_{\operatorname{lin} \mid t=0}=u_{0}
$$

in the case $\dot{\Omega}_{j}=0$, with $\Omega_{j}>0$; see e.g. [19]. It was generalized (still with $\dot{\Omega}_{j}=0$ ) in $[23]$ to a framework where, typically, $\Omega_{j} \in \mathbf{R}$ has no specified sign.

The case of time dependent harmonic potentials with $d=1$ was considered in [16], along with other terms corresponding, for instance, to time dependent magnetic and electric fields. Since the case $d \geqslant 1$ for (3.1) follows by taking the tensor product of the one dimensional case, we shall simply rewrite the results of [16] (and adapt them to our conventions).

Seek formally the solution to (3.1) as

$$
u_{\operatorname{lin}}(t, x)=\left(\prod_{j=1}^{d} \frac{1}{2 i \pi \mu_{j}(t)}\right)^{1 / 2} \int_{\mathbf{R}^{d}} e^{\frac{i}{2} \phi(t, x, y)} u_{0}(y) d y,
$$

where

$$
\phi(t, x, y)=\sum_{j=1}^{d}\left(\alpha_{j}(t) x_{j}^{2}+2 \beta_{j}(t) x_{j} y_{j}+\gamma_{j}(t) y_{j}^{2}+2 \delta_{j}(t) x_{j}+2 \epsilon_{j}(t) y_{j}\right)+\theta(t),
$$

and all the functions of time involved in this formula are real-valued. For instance, when $\Omega=0$, we have $\mu(t)=t, \alpha=\beta=\gamma=1 / t$ and $\delta=\epsilon=\theta=0$ : the convergence $u_{\text {lin }}(t) \rightarrow u_{0}$ as $t \rightarrow 0$ is recovered (at least formally) by applying the stationary phase 
formula. Note that in view of the results of D. Fujiwara [20, 21], we know that there exists $\eta>0$ such that for $|t|<\eta$, the solution to (3.1) can be expressed as

$$
u_{\operatorname{lin}}(t, x)=\frac{1}{(2 i \pi t)^{d / 2}} \int_{\mathbf{R}^{d}} e^{i \varphi(t, x, y)} a(t, x, y) u_{0}(y) d y,
$$

where $a(0, x, y)=1, \partial_{x}^{\alpha} \partial_{y}^{\beta} a \in L^{\infty}(]-\eta, \eta\left[\times \mathbf{R}^{d} \times \mathbf{R}^{d}\right)$ for all $\alpha, \beta \in \mathbf{N}^{d}$, and

$$
\varphi(t, x, y)=\frac{|x-y|^{2}}{2 t}+t \xi(t, x, y)
$$

with $\partial_{x}^{\alpha} \partial_{y}^{\beta} \xi \in L^{\infty}(]-\eta, \eta\left[\times \mathbf{R}^{d} \times \mathbf{R}^{d}\right)$ as soon as $|\alpha+\beta| \geqslant 2$.

Applying the differential operator $i \partial_{t}+\frac{1}{2} \Delta$ to (3.2), and identifying the terms (in $\left.x_{j}^{2}, x_{j} y_{j} \ldots\right)$ in $(3.1)$, we find:

$$
\begin{array}{rll}
x_{j}^{2}: & \dot{\alpha}_{j}+\alpha_{j}^{2}+\Omega_{j}=0 ; & x_{j} y_{j}: \quad \dot{\beta}_{j}+\alpha_{j} \beta_{j}=0 . \\
y_{j}^{2}: & \dot{\gamma}_{j}+\beta_{j}^{2}=0 ; & x_{j}: \quad \dot{\delta}_{j}+\alpha_{j} \delta_{j}=0 . \\
y_{j}: & \dot{\epsilon}_{j}+\beta_{j} \delta_{j}=0 ; & \operatorname{Im}(\mathbf{C}): \quad \dot{\mu}_{j}=\alpha_{j} \mu_{j} . \\
\operatorname{Re}(\mathbf{C}): & \dot{\theta}+\sum_{j=1}^{d} \delta_{j}^{2}=0 . &
\end{array}
$$

We infer that $\mu_{j}$ is given by

$$
\ddot{\mu}_{j}+\Omega_{j}(t) \mu_{j}=0 ; \quad \mu_{j}(0)=0, \quad \dot{\mu}_{j}(0)=1 .
$$

We also have

$$
\alpha_{j}=\frac{\dot{\mu}_{j}}{\mu_{j}}
$$

Note that as in the standard cases $\left(\dot{\Omega}_{j}=0\right), \alpha_{j}(t) \sim 1 / t$ as $t \rightarrow 0$. For $\beta_{j}$, we have

$$
\dot{\beta}_{j}+\frac{\dot{\mu}_{j}}{\mu_{j}} \beta_{j}=0 \text {, hence } \beta_{j}(t)=\frac{C}{\mu_{j}(t)},
$$

and the stationary phase formula (as $t \rightarrow 0$ ) yields $C=-1$. We also find

$$
\gamma_{j}(t)=\frac{1}{\mu_{j}(t) \dot{\mu}_{j}(t)}-\int_{0}^{t} \frac{\Omega_{j}(\tau)}{\left(\dot{\mu}_{j}(\tau)\right)^{2}} d \tau .
$$

Since $\delta_{j}(0)=\epsilon_{j}(0)=\theta(0)=0$, we have $\delta_{j}=\epsilon_{j}=\theta_{j} \equiv 0$.

REMARK 3.1. The case of the usual harmonic potential $\left(\Omega_{j}=1\right)$ shows that singularities may be present in the fundamental solutions for positive times, corresponding to the zeroes of $\mu_{j}$; see e.g. [17, 24, 39, 40].

REMARK 3.2. The dispersive properties associated to (3.1) are measured by the $\mu_{j}$ 's. We will see for instance that if $\Omega_{j} \leqslant 0$ for all $j$, then global in time Strichartz estimates are available, as in the case $\Omega_{j}=0$. To summarize, we have:

Lemma 3.3. Let $d \geqslant 1$, and $\Omega_{j} \in C(\mathbf{R} ; \mathbf{R})$ be locally Lipschitz. There exists $T>0$ such that for $u_{0} \in \mathcal{S}\left(\mathbf{R}^{d}\right)$, the solution to (3.1) is given, for $|t|<T$, by:

$$
u_{\operatorname{lin}}(t, x)=\left(\prod_{j=1}^{d} \frac{1}{2 i \pi \mu_{j}(t)}\right)^{1 / 2} \int_{\mathbf{R}^{d}} e^{\frac{i}{2} \sum_{j=1}^{d}\left(\alpha_{j}(t) x_{j}^{2}+2 \beta_{j}(t) x_{j} y_{j}+\gamma_{j}(t) y_{j}^{2}\right)} u_{0}(y) d y,
$$


where

$$
\begin{aligned}
& \ddot{\mu}_{j}+\Omega_{j}(t) \mu_{j}=0 ; \quad \mu_{j}(0)=0, \quad \dot{\mu}_{j}(0)=1 \\
& \alpha_{j}=\frac{\dot{\mu}_{j}}{\mu_{j}} ; \quad \beta_{j}=-\frac{1}{\mu_{j}} ; \quad \gamma_{j}(t)=\frac{1}{\mu_{j}(t) \dot{\mu}_{j}(t)}-\int_{0}^{t} \frac{\Omega_{j}(\tau)}{\left(\dot{\mu}_{j}(\tau)\right)^{2}} d \tau .
\end{aligned}
$$

REMARK 3.4. The fact that the quadratic potential has no rectangle term is not necessary in order to get such a result. If we consider

$$
i \partial_{t} u_{\mathrm{lin}}+\frac{1}{2} \Delta u_{\mathrm{lin}}=\frac{1}{2}\langle M(t) x, x\rangle u_{\mathrm{lin}},
$$

where $M(t)$ is a (time dependent) symmetric matrix, then a similar formula is available. Of course, the formula is more involved, and since it does not really bring new information, we do not carry out the computation here.

3.2. Some consequences. In this paragraph, we assume that the functions $\Omega_{j}$ are bounded. This assumption was discussed in Example 2.4.

As a consequence of the boundedness of $\Omega_{j}$, we infer a uniform local bound from below for the functions $\mu_{j}$. It follows from the growth of the functions $\mu_{j}$ 's, which is at most exponential:

Lemma 3.5. Assume that for all $j \in\{1, \ldots, d\}, \Omega_{j} \in C(\mathbf{R} ; \mathbf{R})$ is locally Lipschitz and bounded. For $s \in \mathbf{R}$, define $\mu_{j}^{s}$ and $\nu_{j}^{s}$ as the solutions to

$$
\begin{array}{lll}
\ddot{\mu}_{j}^{s}+\Omega_{j}(t) \mu_{j}^{s}=0 & ; \quad \mu_{j}^{s}(s)=0, & \dot{\mu}_{j}^{s}(s)=1 . \\
\ddot{\nu}_{j}^{s}+\Omega_{j}(t) \nu_{j}^{s}=0 & ; \quad \nu_{j}^{s}(s)=1, & \dot{\nu}_{j}^{s}(s)=0 .
\end{array}
$$

There exists $C>0$ independent of $s \in \mathbf{R}$ such that

$$
\left|\mu_{j}^{s}(t)\right|+\left|\dot{\mu}_{j}^{s}(t)\right|+\left|\nu_{j}^{s}(t)\right|+\left|\dot{\nu}_{j}^{s}(t)\right| \leqslant C e^{C|t-s|}, \quad \forall t \in \mathbf{R} .
$$

Proof. Introduce $f_{j}^{s}(t)=\left|\dot{\mu}_{j}^{s}(t)\right|+\left|\mu_{j}^{s}(t)\right|$. We have

$$
\begin{aligned}
\dot{f}_{j}^{s}(t) & \leqslant\left|\ddot{\mu}_{j}^{s}(t)\right|+\left|\dot{\mu}_{j}^{s}(t)\right|=\left|\Omega_{j}(t) \mu_{j}^{s}(t)\right|+\left|\dot{\mu}_{j}^{s}(t)\right| \\
& \leqslant|| \Omega_{j} \|_{L^{\infty}}\left|\mu_{j}^{s}(t)\right|+\left|\dot{\mu}_{j}^{s}(t)\right| \lesssim f_{j}^{s}(t) .
\end{aligned}
$$

Gronwall's Lemma yields, since $f_{j}^{s}(s)=1$,

$$
f_{j}^{s}(t) \lesssim e^{C|t-s|},
$$

for some $C>0$ independent of $j, s$ and $t$. The first part of lemma then follows. The second estimate is similar.

In view of the initial data for $\mu_{j}^{s}$ and $\nu_{j}^{s}$, we infer:

Lemma 3.6. Assume that for all $j \in\{1, \ldots, d\}, \Omega_{j} \in C(\mathbf{R} ; \mathbf{R})$ is locally Lipschitz and bounded. There exists $\eta>0$ such that for all $j$, and all $s \in \mathbf{R}$,

$$
\left|\mu_{j}^{s}(t)\right| \geqslant \frac{|t-s|}{2}, \quad \frac{1}{2} \leqslant\left|\nu_{j}^{s}(t)\right| \leqslant \frac{3}{2}, \quad \forall t,|t-s|<\eta,
$$

where $\mu_{j}^{s}$ and $\nu_{j}^{s}$ are given by (3.4) and (3.5), respectively.

This yields a uniform local dispersion in (2.2), and we infer a property which will be crucial in the study of the large time behavior of high Sobolev norms:

Proposition 3.7. Assume that for all $j, \Omega_{j} \in C(\mathbf{R} ; \mathbf{R})$ is locally Lipschitz and bounded. Then Proposition 2.2 remains valid with $T=\infty$. 


\section{Generalized lens transform}

4.1. The formula. It was noticed in [25] that in the case of the $L^{2}$-critical nonlinearity $(\sigma=2 / d)$, an explicit change of unknown function makes it possible to add or remove an isotropic harmonic potential: if $v$ solves

$$
i \partial_{t} v+\frac{1}{2} \Delta v=\lambda|v|^{4 / d} v \quad ; \quad v_{\mid t=0}=u_{0},
$$

where $\lambda \in \mathbf{R}$, then $u$, given for $|t|<\pi /(2 \omega)$ by the lens transform

$$
u(t, x)=\frac{1}{(\cos (\omega t))^{d / 2}} v\left(\frac{\tan (\omega t)}{\omega}, \frac{x}{\cos (\omega t)}\right) e^{-i \frac{\omega}{2}|x|^{2} \tan (\omega t)}
$$

solves

$$
i \partial_{t} u+\frac{1}{2} \Delta u=\frac{\omega^{2}}{2}|x|^{2} u+\lambda|u|^{4 / d} u ; \quad u_{\mid t=0}=u_{0} .
$$

See also $[30,5,35]$. Note that the change for the time variable is locally, not globally, invertible. The case of a repulsive harmonic potential,

$$
i \partial_{t} u+\frac{1}{2} \Delta u=-\frac{\omega^{2}}{2}|x|^{2} u+\lambda|u|^{4 / d} u ; \quad u_{\mid t=0}=u_{0},
$$

is obtained by replacing $\omega$ by $i \omega$. A formula similar to (4.2) follows, where the trigonometric functions are replaced by hyperbolic functions (and the discussion on the time interval becomes different); see [6]. A heuristic way to understand why this approach works only in the case of isotropic potentials is that even though there would be a "natural" candidate to change the space variable in the anisotropic case, there is no satisfactory candidate to change the time variable.

The lens transform can be generalized to the case of (1.2) provided that the potential is isotropic in the sense that $\Omega_{j}(t)=\Omega(t)$ is independent of $j$. Seek an extension of (4.2) of the form

$$
u(t, x)=\frac{1}{b(t)^{d / 2}} v\left(\zeta(t), \frac{x}{b(t)}\right) e^{\frac{i}{2} a(t)|x|^{2}},
$$

with $a, b, \zeta$ real-valued,

$$
b(0)=1 ; \quad a(0)=\zeta(0)=0 .
$$

Suppose also that $v$ solves a more general non-autonomous equation

$$
i \partial_{t} v+\frac{1}{2} \Delta v=H(t)|v|^{2 \sigma} v ; \quad v_{\mid t=0}=u_{0} .
$$

We want $u$ to solve

$$
i \partial_{t} u+\frac{1}{2} \Delta u=\frac{1}{2} \Omega(t)|x|^{2} u+h(t)|u|^{2 \sigma} u ; \quad u_{\mid t=0}=u_{0} .
$$

Apply the Schrödinger differential operator to the formula (4.3), and identify the terms so that $u$ solves (4.6). We find:

$$
\dot{b}=a b ; \quad \dot{a}+a^{2}+\Omega=0 ; \quad \dot{\zeta}=\frac{1}{b^{2}} ; \quad b(t)^{d \sigma-2} H(\zeta(t))=h(t) .
$$


Introduce the solution to

$$
\left\{\begin{array}{lll}
\ddot{\mu}+\Omega(t) \mu=0 & ; \quad \mu(0)=0, & \dot{\mu}(0)=1 . \\
\ddot{\nu}+\Omega(t) \nu=0 & ; \quad \nu(0)=1, & \dot{\nu}(0)=0 .
\end{array}\right.
$$

Note that since the Wronskian of $\mu$ and $\nu$ is constant, we have $\dot{\mu} \nu-\mu \dot{\nu}=1$ for all time. This relation extends the identities $\cos ^{2} t+\sin ^{2} t=1$ and $\cosh ^{2} t-\sinh ^{2} t=1$. In view of (4.4), we infer:

$$
a=\frac{\dot{\nu}}{\nu} ; \quad b=\nu ; \quad \zeta=\frac{\mu}{\nu} .
$$

Note that $\zeta$ is locally invertible since $\zeta(0)=0$ and

$$
\dot{\zeta}=\frac{1}{b^{2}}=\frac{1}{\nu^{2}} \text {, hence } \dot{\zeta}(0)=1 \text {. }
$$

Therefore, the lens transform is locally invertible. Moreover, since $b(0)=\nu(0)=1$, we can write, locally in time,

$$
H(t)=b\left(\zeta^{-1}(t)\right)^{2-d \sigma} h\left(\zeta^{-1}(t)\right)=\nu\left(\left(\frac{\mu}{\nu}\right)^{-1}(t)\right)^{2-d \sigma} h\left(\left(\frac{\mu}{\nu}\right)^{-1}(t)\right) .
$$

Proposition 4.1. Let $v$ solve

$$
i \partial_{t} v+\frac{1}{2} \Delta v=H(t)|v|^{2 \sigma} v \quad ; \quad v_{\mid t=0}=u_{0} .
$$

Let $\Omega \in C(\mathbf{R} ; \mathbf{R})$. There exists $T>0$ such that the following holds. Define $u$ by

$$
u(t, x)=\frac{1}{\nu(t)^{d / 2}} v\left(\frac{\mu(t)}{\nu(t)}, \frac{x}{\nu(t)}\right) e^{i \frac{\dot{\nu}(t)}{\nu(t)} \frac{|x|^{2}}{2}}, \quad|t| \leqslant T,
$$

where $(\mu, \nu)$ is given by (4.7). Then for $|t|<\mu(T) / \nu(T)$, u solves

$$
i \partial_{t} u+\frac{1}{2} \Delta u=\frac{1}{2} \Omega(t)|x|^{2} u+h(t)|u|^{2 \sigma} u ; \quad u_{\mid t=0}=u_{0},
$$

where $h(t)=\nu(t)^{d \sigma-2} H(\mu(t) / \nu(t))$.

REMARK 4.2. We do not require $\Omega$ to be locally Lipschitz: all we need is the local existence of a $C^{2}$ solution to (4.7), so we can rely on Peano existence theorem.

4.2. Proof of Proposition 1.10. We assume in this paragraph that the nonlinearity is focusing: $\lambda<0$. By homogeneity, we can assume $\lambda=-1$. It is well known that the equation

$$
i \partial_{t} v+\frac{1}{2} \Delta v=-|v|^{4 / d} v
$$

possesses solutions which blow up in finite time, with different possible rates (see e.g. $[3,13,29,33,34]$ and references therein).

By adapting Proposition 4.1 to isolate the initial time $t=0$, we see that the lens transform maps a solution to (4.8) which blows up at time $t=0$ to a solution to

$$
i \partial_{t} u+\frac{1}{2} \Delta u=\frac{1}{2} \Omega(t)|x|^{2} u-|u|^{4 / d} u
$$

which blows up at time $t=0$. Note that the blow-up rate is not altered by the lens transform, since $\nu(t) \approx 1$ and $\mu(t) / \nu(t) \approx t$ as $t \rightarrow 0$. 
Typically, consider the (unstable) minimal mass blow-up solution to (4.8):

$$
v(t, x)=\frac{1}{t^{d / 2}} Q\left(\frac{x}{t}\right) e^{i \frac{|x|^{2}}{2 t}-\frac{i}{t}},
$$

where $Q$ is the ground state, defined as the unique positive radial solution to

$$
-\frac{1}{2} \Delta Q+Q=Q^{1+4 / d}
$$

The lens transform yields a corresponding blow-up solution to (4.9) given by

$$
u(t, x)=\frac{1}{\mu(t)^{d / 2}} Q\left(\frac{x}{\mu(t)}\right) e^{i \frac{\dot{\mu}(t)}{\mu(t)} \frac{|x|^{2}}{2}-i \frac{\nu(t)}{\mu(t)}} .
$$

To our knowledge, this gives the first example of an explicit blow-up solution in the presence of a time-dependent external potential.

Note that we have considered the explicit case of minimal mass blow-up solutions for convenience. Any blow-up solution for (4.8) gives rise to a blow-up solution for (4.9), with the same blow-up rate.

Note also that without the extra assumption on $\Omega$, the Sobolev norms of $u$ may have an arbitrary growth rate as $t \rightarrow \infty$.

EXAmple 4.3. Consider $\mu(t)=\exp \left(1-e^{t}\right)-\exp \left(1-e^{2 t}\right)$ (which satisfies $\mu(0)=0$ and $\dot{\mu}(0)=1)$. Then the growth of Sobolev norms of the function u given by the above formula is given by a double exponential in time, since

$$
\|u(t)\|_{H^{s}} \underset{t \rightarrow+\infty}{\sim} \frac{C_{s}}{|\mu(t)|^{s}} .
$$

To determine the corresponding function $\Omega$, we compute

$$
\ddot{\mu}(t)=\left(e^{2 t}-e^{t}\right)\left(\exp \left(1-e^{t}\right)-4 \exp \left(1-e^{2 t}\right)\right),
$$

and therefore

$$
\Omega(t)=\frac{\exp \left(1-e^{t}\right)-4 \exp \left(1-e^{2 t}\right)}{\exp \left(1-e^{t}\right)-\exp \left(1-e^{2 t}\right)}\left(e^{t}-e^{2 t}\right) .
$$

We note that $\Omega(t) \sim-e^{2 t}$ as $t \rightarrow+\infty$; the harmonic potential is repulsive $(\Omega<0)$, and becomes exponentially stronger as time increases.

\section{Vector fields}

The aim of this paragraph is to show that there exists vector fields which may be useful to study the nonlinear equation (1.2), in the same spirit as in $[6,8]$. Consider the solutions to

$$
\begin{cases}\ddot{\mu}_{j}+\Omega_{j}(t) \mu_{j}=0 & ; \quad \mu_{j}(0)=0, \quad \dot{\mu}_{j}(0)=1 \\ \ddot{\nu}_{j}+\Omega_{j}(t) \nu_{j}=0 & ; \quad \nu_{j}(0)=1, \quad \dot{\nu}_{j}(0)=0\end{cases}
$$

We define

$$
\begin{aligned}
& A_{j}=\dot{\mu}_{j} x_{j}+i \mu_{j} \partial_{j}=i \mu_{j} e^{i \frac{x_{j}^{2}}{2} \frac{\dot{\mu}_{j}}{\mu_{j}}} \partial_{j}\left(e^{-i \frac{x_{j}^{2}}{2} \frac{\dot{\mu}_{j}}{\mu_{j}}} \cdot\right)=i \mu_{j} e^{i \sum_{k} \frac{x_{k}^{2}}{2} \frac{\mu_{k}}{\mu_{k}}} \partial_{j}\left(e^{-i \sum_{k} \frac{x_{k}^{2}}{2} \frac{\dot{\mu}_{k}}{\mu_{k}}} \cdot\right) \\
& B_{j}=\dot{\nu}_{j} x_{j}+i \nu_{j} \partial_{j}=i \nu_{j} e^{i \frac{x_{j}^{2}}{2} \frac{\dot{\nu}_{j}}{\nu_{j}}} \partial_{j}\left(e^{-i \frac{x_{j}^{2}}{2} \frac{\dot{\nu}_{j}}{\nu_{j}}} \cdot\right)=i \nu_{j} e^{i \sum_{k} \frac{x_{k}^{2}}{2} \frac{\dot{\nu}_{k}}{\nu_{k}}} \partial_{j}\left(e^{-i \sum_{k} \frac{x_{k}^{2}}{2} \frac{\dot{\nu}_{k}}{\nu_{k}}} \cdot\right)
\end{aligned}
$$


Note that the last two expressions for $A$ or $B$ show that $A$ and $B$ act on gauge invariant nonlinearities like derivatives; the modulus ignores the multiplication by the exponential.

EXAMPLE $5.1\left(\dot{\Omega}_{j}=0\right)$. When $\Omega_{j}=0, B_{j}=i \partial_{j}$ and $A_{j}=x_{j}+i t \partial_{j}$, which are Heisenberg derivatives commonly used in the theory of nonlinear Schrödinger equations (see e.g. [13]). When $\Omega_{j}=\omega_{j}^{2}>0, A_{j}=x_{j} \cos \left(\omega_{j} t\right)+i \frac{\sin \left(\omega_{j} t\right)}{\omega_{j}} \partial_{j}$ and $B_{j}=$ $\omega_{j} x_{j} \sin \left(\omega_{j} t\right)+i \cos \left(\omega_{j} t\right) \partial_{j}$, and hence we recover classical Heisenberg derivatives (see e.g. [18]). In these two cases (as well as in the case $\Omega_{j}=-\omega_{j}^{2}<0$ ), we have

$$
A_{j}=U_{V}(t) x_{j} U_{V}(-t) ; \quad B_{j}=U_{V}(t) i \partial_{j} U_{V}(-t),
$$

where $U_{V}(t)=\exp \left(-i t\left(-\frac{1}{2} \Delta+V(x)\right)\right), V(x)=\sum_{k=1}^{d} \Omega_{k} x_{k}^{2}$.

More generally, consider $\dot{\eta}_{j} x_{j}+i \eta_{j} \partial_{j}$. We check that this operator commutes with the linear operator

$$
i \partial_{t}+\frac{1}{2} \Delta-\frac{1}{2} \sum_{k=1}^{d} \Omega_{k}(t) x_{k}^{2}
$$

if and only if $\eta_{j}$ satisfies $\ddot{\eta}_{j}+\Omega_{j} \eta_{j}=0$, in view of the identity

$$
\begin{aligned}
{\left[i \partial_{t}+\frac{1}{2} \Delta-\frac{1}{2} \sum_{k=1}^{d} \Omega_{k}(t) x_{k}^{2}, \dot{\eta}_{j} x_{j}+i \eta_{j} \partial_{j}\right] } & =\left[i \partial_{t}+\frac{1}{2} \partial_{j}^{2}-\frac{1}{2} \Omega_{j}(t) x_{j}^{2}, \dot{\eta}_{j} x_{j}+i \eta_{j} \partial_{j}\right] \\
& =i \ddot{\eta}_{j} x_{j}-\dot{\eta}_{j} \partial_{j}+\dot{\eta}_{j} \partial_{j}+i \eta_{j} \Omega_{j} x_{j} .
\end{aligned}
$$

REMARK 5.2. This computation could be extended to the case where the center of the harmonic potential depends on time:

$$
i \partial_{t} u+\frac{1}{2} \Delta u=\frac{1}{2} \sum_{k=1}^{d} \Omega_{k}(t)\left(x_{k}-c_{k}(t)\right)^{2} u .
$$

Replacing $\dot{\eta}_{j} x_{j}+i \eta_{j} \partial_{j}$ with $\dot{\eta}_{j}\left(x_{j}-y_{j}(t)\right)+i \eta_{j} \partial_{j}$, we can repeat the above computation and check that the two operators commute if and only if $\ddot{\eta}_{j}+\Omega_{j} \eta_{j}=0$ and $\ddot{\eta}_{j} y_{j}+\dot{\eta}_{j} \dot{y}_{j}+\eta_{j} \Omega_{j} c_{j}=0$. We choose not to investigate this case in detail here.

To show that the $\Sigma$-norm of $u$ is related to the $L^{2}$-norms of $A_{j} u$ and $B_{j} u$, write

$$
\left(\begin{array}{c}
A_{j} \\
B_{j}
\end{array}\right)=M_{j}\left(\begin{array}{c}
x_{j} \\
i \partial_{j}
\end{array}\right), \quad \text { where } M_{j}=\left(\begin{array}{cc}
\dot{\mu}_{j} & \mu_{j} \\
\dot{\nu}_{j} & \nu_{j}
\end{array}\right) .
$$

We note that the determinant of $M_{j}$ is the Wronskian of $\mu_{j}$ and $\nu_{j}$ :

$$
\operatorname{det} M_{j}=\nu_{j} \dot{\mu}_{j}-\mu_{j} \dot{\nu}_{j} \equiv 1
$$

Therefore

$$
\left(\begin{array}{c}
x_{j} \\
i \partial_{j}
\end{array}\right)=\left(\begin{array}{cc}
\nu_{j} & -\mu_{j} \\
-\dot{\nu}_{j} & \dot{\mu}_{j}
\end{array}\right)\left(\begin{array}{c}
A_{j} \\
B_{j}
\end{array}\right)
$$


We shall use these vector fields in the isotropic case, where they provide a priori estimates:

$$
i \partial_{t} u+\frac{1}{2} \Delta u=\frac{1}{2} \Omega(t)|x|^{2} u+\lambda|u|^{2 \sigma} u .
$$

Since $A$ commutes with the linear part of (5.3) and acts on gauge invariant nonlinearities like a gradient, we have

$$
\frac{1}{2} \frac{d}{d t}\|A u\|_{L^{2}}^{2}=\lambda \sigma \operatorname{Im} \int_{\mathbf{R}^{d}}|u|^{2 \sigma-2} u^{2}(\overline{A u})^{2} .
$$

Expanding $(A u)^{2}$, we eventually obtain

$$
\begin{aligned}
& \frac{d}{d t}\left(\frac{1}{2}\|A u\|_{L^{2}}^{2}+\frac{\lambda \mu^{2}}{\sigma+1}\|u\|_{L^{2 \sigma+2}}^{2 \sigma+2}\right)=\frac{\lambda}{\sigma+1} \mu \dot{\mu}(2-d \sigma)\|u\|_{L^{2 \sigma+2}}^{2 \sigma+2}, \\
& \frac{d}{d t}\left(\frac{1}{2}\|B u\|_{L^{2}}^{2}+\frac{\lambda \nu^{2}}{\sigma+1}\|u\|_{L^{2 \sigma+2}}^{2 \sigma+2}\right)=\frac{\lambda}{\sigma+1} \nu \dot{\nu}(2-d \sigma)\|u\|_{L^{2 \sigma+2}}^{2 \sigma+2} .
\end{aligned}
$$

These evolution laws are the analogue of the pseudo-conformal conservation law (see [8] for the case $\dot{\Omega}=0$ ). They will allow us to infer scattering results in the case $\Omega \leqslant 0$, $\lambda \geqslant 0(\S 6.3)$.

\section{Growth of higher order Sobolev norms and momenta}

6.1. The linear case. In this paragraph, we assume $\lambda=0$. We recall that in general, Mehler's formula is valid only locally in time, since singularities may appear in the fundamental solution; see e.g. [17, 24, 39, 40]. To understand the long time behavior of the solution $u_{\text {lin }}$ to (3.1), one may use Egorov's Theorem (see e.g. [4]). Since we deal with a time-dependent potential, modifications would be needed in Egorov's Theorem, and we follow instead another strategy to have some estimates in the linear case (instead of an exact asymptotic behavior, as Egorov's Theorem would give us). This approach is based on the vector fields introduced in $\S 5$.

We remark that since the $L^{2}$-norm of $u_{\text {lin }}$ does not depend on time, and since the operators $A_{j}$ and $B_{j}$ introduced in $\S 5$ commute with Equation (3.1), the $L^{2}$-norm of $A_{j_{1}} B_{j_{2}} \ldots A_{j_{k}} u_{\text {lin }}$ is constant for whichever combination of these vector fields. In view of (5.2) we infer, for $k \in \mathbf{N}$,

$$
\left\||x|^{k} u_{\operatorname{lin}}(t)\right\|_{L^{2}}+\left\|u_{\operatorname{lin}}(t)\right\|_{H^{k}} \lesssim \sum_{j=1}^{d}\left(\left|\mu_{j}(t)\right|^{k}+\left|\nu_{j}(t)\right|^{k}\right) .
$$

Lemma 3.5 shows that if $\Omega_{j} \in C(\mathbf{R} ; \mathbf{R})$ is locally Lipschitz and bounded, then the above quantity grows at most exponentially in time. By Proposition 3.7, we conclude that $(E x p)_{k}$ is satisfied for all $k$, provided $u_{0}$ is sufficiently smooth and localized. We recall that the case $\Omega_{j}=-1$ shows that the exponential growth may occur, and that in $(E x p)_{k}$, the constant $C$ must be expected to depend on $k(C=k$ when $\Omega=-1$ is sharp).

6.2. The $L^{2}$-subcritical case.

Lemma 6.1. Let $\sigma, k \in \mathbf{N}$, with $\sigma \leqslant 2 / d, \Omega_{j} \in C(\mathbf{R} ; \mathbf{R})$ be locally Lipschitz and bounded, and

$$
u_{0} \in H^{k}\left(\mathbf{R}^{d}\right) \text {, with }|x|^{k} u_{0} \in L^{2}\left(\mathbf{R}^{d}\right)
$$


Suppose that there exists $f \in C\left(\mathbf{R}_{+} ; \mathbf{R}_{+}\right)$with $f(0)=0$ such that

$$
\|u\|_{L^{\theta}\left([s, s+\tau] ; L^{q}\right)} \leqslant f(\tau), \quad \forall s, \tau \in \mathbf{R},
$$

where

$$
q=2 \sigma+2 ; \quad \theta=\frac{2 \sigma(2 \sigma+2)}{2-(d-2) \sigma} .
$$

Then the solution to (1.2) satisfies $(\text { Exp })_{k}$.

Proof. The first step consists in resuming the computations carried out in the proof of Proposition 2.5, in the case $k=0$. The case $k \geqslant 1$ will follow by induction (recall that the constant $C$ in the exponential growth must be expected to depend on $k)$.

Case $k=0$. Let us pretend that the $L^{2}$-norm of $u$ is not conserved, to simplify the induction. Resuming the same numerology as in the proof of Proposition 2.5, Strichartz estimates yield, for all $t \in \mathbf{R}$ and $\tau>0$,

$$
\begin{aligned}
\|u\|_{L^{p}\left([t, t+\tau] ; L^{q}\right) \cap L^{\infty}\left([t, t+\tau] ; L^{2}\right)} & \lesssim\|u(t)\|_{L^{2}}+\|u\|_{L^{\theta}\left([t, t+\tau] ; L^{q}\right)}^{2 \sigma}\|u\|_{L^{p}\left([t, t+\tau] ; L^{q}\right)} \\
& \lesssim\|u(t)\|_{L^{2}}+f(\tau)^{2 \sigma}\|u\|_{L^{p}\left([t, t+\tau] ; L^{q}\right)} .
\end{aligned}
$$

Fix $\tau \ll 1$ once and for all so the last term of the right hand side can be absorbed by the left hand side, up to doubling the estimation constant. At every increment of time of length $\tau$, the $L^{2}$ norm is multiplied (at most) by some fixed constant $C$. This implies that it grows at most exponentially. Using Strichartz estimates again, we conclude that $(E x p)_{0}$ is satisfied (and actually, $(A l g)_{0}$ is also true).

Case $k \geqslant 1$. For $k \geqslant 1$, suppose that $(\operatorname{Exp})_{k-1}$ is satisfied. To avoid a lengthy presentation, we denote by $w_{\ell}$ the family of combinations of $\alpha$ momenta and $\beta$ space derivatives of $u$, with $|\alpha|+|\beta|=\ell\left(w_{0}=u\right)$. We have, rather formally,

$$
i \partial_{t} w_{k}+\frac{1}{2} \Delta w_{k}=\frac{1}{2} \sum_{j=1}^{d} \Omega_{j}(t) x_{j}^{2} w_{k}+\mathrm{V}\left(u, w_{k}\right)+F+L\left(w_{k}\right),
$$

where $\mathrm{V}$ is homogeneous of degree $2 \sigma$ with respect to its first argument, $\mathbf{R}$-linear with respect to its second argument, $F$ satisfies the pointwise estimate

$$
|F| \lesssim \sum_{0 \leqslant \ell_{j} \leqslant k-1}\left|w_{\ell_{1}}\right| \ldots\left|w_{\ell_{2 \sigma+1}}\right|
$$

where the sums carries over combinations such that in addition $\sum \ell_{j}=k(F=0$ in the case $k=1$ ), and $L$ is linear with respect to its argument. A word of explanation is needed about $L$ : this term stems from the fact that $x$ and $\nabla$ do not commute with the linear part of the equation. One might argue that we could proceed as in the linear case, and use repeatedly the vector fields $A_{j}$ and $B_{j}$. The problem is that even though $A_{j}$ and $B_{j}$ act on gauge invariant nonlinearities like derivatives, this is not so, for instance, for $A_{j} B_{j}$ (the phases do not cancel in the factored formula). We might use the operators $A_{j_{1}} \ldots A_{j_{k}}$ and $B_{j_{1}} \ldots B_{j_{k}}$, but this does not suffice to recover the momenta and derivatives of $u$ since "rectangle" terms (like $A_{j} B_{j}$ ) would be needed. 
We proceed in the same spirit as in the case $k=0$ :

$$
\begin{gathered}
\left\|w_{k}\right\|_{L^{p}\left([t, t+\tau] ; L^{q}\right) \cap L^{\infty}\left([t, t+\tau] ; L^{2}\right)} \lesssim\left\|w_{k}(t)\right\|_{L^{2}}+\|u\|_{L^{\theta}\left([t, t+\tau] ; L^{q}\right)}^{2 \sigma}\left\|w_{k}\right\|_{L^{p}\left([t, t+\tau] ; L^{q}\right)} \\
+\sum_{0 \leqslant \ell_{j} \leqslant k-1}\left\|w_{\ell_{1}}\right\|_{L^{\theta}\left([t, t+\tau] ; L^{q}\right)} \cdots\left\|w_{\ell_{2 \sigma}}\right\|_{L^{\theta}\left([t, t+\tau] ; L^{q}\right)}\left\|w_{\ell_{2 \sigma+1}}\right\|_{L^{p}\left([t, t+\tau] ; L^{q}\right)} \\
+\left\|L\left(w_{k}\right)\right\|_{L^{1}\left([t, t+\tau] ; L^{2}\right)}
\end{gathered}
$$

Fixing $\tau \ll 1$ independent of $t \in \mathbf{R}$, the second term of the right hand side is absorbed by the left hand side. The sum is treated thanks to $(\operatorname{Exp})_{k-1}$. We notice that since $\sigma \leqslant 2 / d$, we have $\theta \leqslant p$, where we recall that $(p, q)$ is admissible: for $1 \leqslant j \leqslant 2 \sigma$,

$$
\left\|w_{\ell_{j}}\right\|_{L^{\theta}\left([t, t+\tau] ; L^{q}\right)} \leqslant \tau^{1 / \theta-1 / p}\left\|w_{\ell_{j}}\right\|_{L^{p}\left([t, t+\tau] ; L^{q}\right)} \lesssim \tau^{1 / \theta-1 / p} e^{C(t+\tau)},
$$

where we have used $(E x p)_{k-1}$. The last term of the sum is estimated similarly.

Finally, the term $L\left(w_{k}\right)$ is handled by the Gronwall's Lemma, and $(\operatorname{Exp})_{k}$ follows.

The proof of Proposition 1.13 in the one-dimensional cubic case follows readily. Since this case is $L^{2}$-subcritical, we have $\theta<p$. Using a Strichartz estimate, we infer, for $s, \tau \in \mathbf{R}$,

$$
\begin{aligned}
\|u\|_{L^{p}\left([s, s+\tau] ; L^{q}\right)} & \leqslant C(p)\left(\left\|u_{0}\right\|_{L^{2}}+\|u\|_{L^{\theta}\left([s, s+\tau] ; L^{q}\right)}^{2 \sigma}\|u\|_{L^{p}\left([s, s+\tau] ; L^{q}\right)}\right) \\
& \leqslant C(p)\left(\left\|u_{0}\right\|_{L^{2}}+\tau^{2 \sigma(1 / \theta-1 / p)}\|u\|_{L^{p}\left([s, s+\tau] ; L^{q}\right)}^{2 \sigma+1}\right),
\end{aligned}
$$

for some $C(p)$ is independent of $s$ and $\tau$, where we have used the conservation of mass. Choosing $\tau$ sufficiently small, a bootstrap argument implies that there exists $C>0$ such that

$$
\|u\|_{L^{p}\left([s, s+\tau] ; L^{q}\right)} \leqslant C, \quad \forall s \in \mathbf{R}, 0<\tau \leqslant \tau_{0} .
$$

Again since $\theta<p$, we conclude that (6.1) is satisfied with $f(\tau)=C \tau^{1 / \theta-1 / p}$.

6.3. Isotropic repulsive potential. We assume $\sigma \geqslant 2 / d$, and $\lambda \geqslant 0$ (defocusing nonlinearity). We show that in the isotropic repulsive case $\Omega_{j}=\Omega \geqslant 0$ (a case where the energy $E$ defined in (1.4) is not a positive functional), the evolution laws derived in $\S 5$ show us that the nonlinearity is negligible for large time, and there is scattering. In this paragraph, we also assume that $\Omega$ is locally Lipschitz, without systematically recalling this assumption. We start with the straightforward result:

Lemma 6.2. Assume $\Omega_{j}(t) \leqslant 0$ for all $t \geqslant 0$. Then the solutions to (5.1) satisfy

$$
\nu_{j}(t) \geqslant 1, \quad \mu_{j}(t) \geqslant t, \quad \dot{\nu}_{j}(t) \geqslant 0, \quad \dot{\mu}_{j}(t) \geqslant 1, \quad \forall t \geqslant 0 .
$$

Remark 6.3. As a consequence of this lemma, Proposition 2.2 remains valid with $T=\infty$, even if $\Omega \leqslant 0$ is not bounded.

We can then prove:

Proposition 6.4. Assume $\Omega_{j}=\Omega$ is independent of $j$, with $\Omega(t) \leqslant 0$ for all $t \geqslant 0$. Let $2 / d \leqslant \sigma(<2 /(d-2)$ if $d \geqslant 3), \lambda \geqslant 0$, and $u_{0} \in \Sigma$. The solution to (1.2) is global in time, and there is scattering:

$$
\exists u_{+} \in \Sigma, \quad\left\|\Psi(t)\left(u(t)-U(t, 0) u_{+}\right)\right\|_{L^{2}} \underset{t \rightarrow+\infty}{\longrightarrow} 0,
$$


for any $\Psi \in\left\{\operatorname{Id}, A_{j}, B_{j}\right\}$, and where $U(t, 0)$ corresponds to the free evolution (3.1).

Proof. Since $\lambda \geqslant 0$ and $\sigma \geqslant 2 / d,(5.4),(5.5)$, and Lemma 6.2 yield

$$
\frac{d}{d t}\left(\frac{1}{2}\|A u\|_{L^{2}}^{2}+\frac{\lambda \mu^{2}}{\sigma+1}\|u\|_{L^{2 \sigma+2}}^{2 \sigma+2}\right) \leqslant 0 ; \quad \frac{d}{d t}\left(\frac{1}{2}\|B u\|_{L^{2}}^{2}+\frac{\lambda \nu^{2}}{\sigma+1}\|u\|_{L^{2 \sigma+2}}^{2 \sigma+2}\right) \leqslant 0 .
$$

We infer a priori bounds for $A u$ and $B u$ in $L^{2}$. Duhamel's formula reads, for $s \in \mathbf{R}$ :

$$
u(t)=U(t, s) u(s)-i \lambda \int_{s}^{t} U(t, \tau)\left(|u|^{2 \sigma} u(\tau)\right) d \tau .
$$

For $\Psi \in\{\operatorname{Id}, A, B\}$, apply $\Psi$ to the above formula:

$$
\Psi(t) u(t)=U(t, s) \Psi(s) u(s)-i \lambda \int_{s}^{t} U(t, \tau) \Psi(\tau)\left(|u|^{2 \sigma} u(\tau)\right) d \tau,
$$

where we have used the fact that $\Psi$ commutes with the linear part of the equation. Since $\Psi$ acts on gauge invariant nonlinearities like a derivative, we have, thanks to Strichartz estimates:

$$
\|\Psi u\|_{L^{p}\left([s, t] ; L^{q}\right) \cap L^{\infty}\left([s, t] ; L^{2}\right)} \lesssim\|\Psi(s) u(s)\|_{L^{2}}+\|u\|_{L^{\theta}\left([s, t] ; L^{q}\right)}^{2 \sigma}\|\Psi u\|_{L^{p}\left([s, t] ; L^{q}\right)},
$$

with the same numerology as in the proof of Proposition 2.5. Since $u, A u$, and $B u$ belong to $L^{\infty}\left(\mathbf{R}_{+} ; L^{2}\left(\mathbf{R}^{d}\right)\right)$, we have

$$
\|u(t)\|_{L^{q}}=\|u(t)\|_{L^{2 \sigma+2}} \lesssim \frac{1}{\langle t\rangle^{d \sigma /(2 \sigma+2)}},
$$

where we have used the factorization formula for $A$ and $B$, the Gagliardo-Nirenberg inequality, and Lemma 6.2. We infer that $u \in L^{\theta}\left(\mathbf{R}_{+} ; L^{q}\right)$ :

$$
\theta \frac{d \sigma}{2 \sigma+2}=\frac{2 d \sigma^{2}}{2-(d-2) \sigma}>1,
$$

since $2 d \sigma^{2}+(d-2) \sigma=2 \sigma(d \sigma-1)+d \sigma>2$. Dividing $\mathbf{R}$ into a finite number of intervals on which the $L^{\theta} L^{q}$-norm of $u$ is small, we infer that $\Psi u \in L^{p}\left(\mathbf{R}_{+} ; L^{q}\right)$. Scattering follows easily:

$$
U(0, t) u(t)=u_{0}-i \lambda \int_{0}^{t} U(0, s)\left(|u|^{2 \sigma} u(s)\right) d s .
$$

For $\widetilde{\Psi} \in\{\mathrm{Id}, \nabla, x\}$, apply $\widetilde{\Psi}$ to the above formula to obtain

$$
\begin{aligned}
\widetilde{\Psi} U(0, t) u(t) & =\widetilde{\Psi} u_{0}-i \lambda \int_{0}^{t} \widetilde{\Psi} U(0, s)\left(|u|^{2 \sigma} u(s)\right) d s \\
& =\widetilde{\Psi} u_{0}-i \lambda \int_{0}^{t} U(0, s) \Psi\left(|u|^{2 \sigma} u(s)\right) d s,
\end{aligned}
$$

where $\Psi=\mathrm{Id}$ if $\widetilde{\Psi}=\mathrm{Id}, \Psi=-i B$ if $\widetilde{\Psi}=\nabla$, and $\Psi=A$ if $\widetilde{\Psi}=x$, respectively. We have

$$
\begin{aligned}
\left\|\widetilde{\Psi} U\left(0, t_{2}\right) u\left(t_{2}\right)-\widetilde{\Psi} U\left(0, t_{1}\right) u\left(t_{1}\right)\right\|_{L^{2}} & \lesssim\left\|\int_{t_{1}}^{t} U(0, s) \Psi\left(|u|^{2 \sigma} u(s)\right) d s\right\|_{L^{\infty}\left(\left[t_{1}, t_{2}\right] ; L^{2}\right)} \\
& \lesssim\left\|\Psi\left(|u|^{2 \sigma} u\right)\right\|_{L^{p^{\prime}}\left(\left[t_{1}, t_{2}\right] ; L^{q^{\prime}}\right)} \\
& \lesssim\|u\|_{L^{\theta}\left(\left[t_{1}, t_{2}\right] ; L^{q}\right)}^{2 \sigma}\|\Psi u\|_{L^{p}\left(\left[t_{1}, t_{2}\right] ; L^{q}\right)} \underset{t_{1}, t_{2} \rightarrow+\infty}{\longrightarrow} 0 .
\end{aligned}
$$


Therefore, $U(0, t) u(t)$ converges to some $u_{+} \in \Sigma$, and the proposition follows.

This result strongly suggests that the solution to the nonlinear equation has the same behavior as the solution to the linear equation as time goes to infinity. It should therefore not be surprising that $(E x p)_{k}$ is satisfied in this case. However, the delicate issue is to measure high order Sobolev norms. To do so, we modify the argument of Lemma 6.1. We will use the operators $A$ and $B$ once, and just once in view of the discussion in the proof of Lemma 6.1.

We have seen in the course of the proof of Proposition 6.4 that $u \in L^{\theta}\left(\mathbf{R} ; L^{q}\right)$ and $\Psi u \in L^{p}\left(\mathbf{R} ; L^{q}\right)$ for $\Psi \in\{\operatorname{Id}, A, B\}$. As announced above, we modify the induction argument of Lemma 6.1: we first apply either $A$ or $B$ to (1.2), and then apply a combination of $x^{\alpha}$ and $\partial_{x}^{\beta}$. We still denote by $w_{\ell}$ the family of combinations of $\alpha$ momenta and $\beta$ space derivatives, now applied to either $A u$ or $B u$, with $|\alpha|+|\beta|=$ $\ell-1$. This will (eventually) not alter the conclusion in view of (5.2) and Lemma 3.5. Despite this small change in the definition of $w_{\ell}$, we still have (6.2) for $k \geqslant 2$ (the case $k \leqslant 1$ is of no interest, since we know that $\Psi u \in L^{p}\left(\mathbf{R} ; L^{q}\right) \cap L^{\infty}\left(\mathbf{R} ; L^{2}\right)$ for $\Psi \in$ $\{\operatorname{Id}, A, B\})$. Resume the key estimate for $w_{k}$ :

$$
\begin{aligned}
& \left\|w_{k}\right\|_{L^{p}\left([t, t+\tau] ; L^{q}\right) \cap L^{\infty}\left([t, t+\tau] ; L^{2}\right)} \\
& \lesssim\left\|w_{k}(t)\right\|_{L^{2}}+\|u\|_{L^{\theta}\left([t, t+\tau] ; L^{q}\right)}^{2 \sigma}\left\|w_{k}\right\|_{L^{p}\left([t, t+\tau] ; L^{q}\right)} \\
& \quad+\sum_{0 \leqslant \ell_{j} \leqslant k-1}\left\|w_{\ell_{1}}\right\|_{L^{\theta}\left([t, t+\tau] ; L^{q}\right)} \ldots\left\|w_{\ell_{2 \sigma}}\right\|_{L^{\theta}\left([t, t+\tau] ; L^{q}\right)}\left\|w_{\ell_{2 \sigma+1}}\right\|_{L^{p}\left([t, t+\tau] ; L^{q}\right)} \\
& \quad+\left\|L\left(w_{k}\right)\right\|_{L^{1}\left([t, t+\tau] ; L^{2}\right)}
\end{aligned}
$$

Fixing $\tau \ll 1$ independent of $t \in \mathbf{R}$, the second term of the right hand side is absorbed by the left hand side. The only difficulty consists in analyzing the sum. We may assume that $\ell_{2 \sigma+1}$ corresponds to the largest value of indices $\ell$. For $1 \leqslant j \leqslant 2 \sigma$, if $\ell_{j} \leqslant k-2$, then we simply estimate

$$
\begin{aligned}
\left\|w_{\ell_{j}}\right\|_{L^{\theta}\left([t, t+\tau] ; L^{q}\right)} & \leqslant \tau^{1 / \theta}\left\|w_{\ell_{j}}\right\|_{L^{\infty}\left([t, t+\tau] ; L^{q}\right)} \lesssim \tau^{1 / \theta}\left\|w_{\ell_{j}}\right\|_{L^{\infty}\left([t, t+\tau] ; H^{1}\right)} \\
& \lesssim \tau^{1 / \theta}\left\|w_{\ell_{j}+1}\right\|_{L^{\infty}\left([t, t+\tau] ; L^{2}\right)} \lesssim e^{C(t+\tau)}
\end{aligned}
$$

where we have used $(\operatorname{Exp})_{k-1}$. So the only case we have to examine is when $\ell_{2 \sigma+1}=$ $k-1=\ell_{j_{0}}$ for some $1 \leqslant j_{0} \leqslant 2 \sigma$. Note that since $\sum \ell_{j}=k$, this may happen only when $k=2$. In that case, we can assume that the term $w_{\ell_{2 \sigma+1}}$ is of the form $A u$ or $B u$ (a term which is $\left.L^{p}\left(\mathbf{R} ; L^{q}\right)\right)$, and estimate as above

$$
\left\|w_{\ell_{j_{0}}}\right\|_{L^{\theta}\left([t, t+\tau] ; L^{q}\right)} \lesssim \tau^{1 / \theta}\left\|w_{2}\right\|_{L^{\infty}\left([t, t+\tau] ; L^{2}\right)} .
$$

The corresponding term in the sum can therefore be absorbed by the left hand side (like V). In the other cases, we estimate $\left\|w_{\ell_{2 \sigma+1}}\right\|_{L^{p}\left([t, t+\tau] ; L^{q}\right)}$ thanks to $(\operatorname{Exp})_{k-1}$. Having examined all the possibilities, we conclude that $(E x p)_{k}$ is satisfied. Note that Proposition 6.4 suggests that the large time behavior of higher (weighted) Sobolev norms of $u$ is the same as in the linear case, so the exponential growth is sharp in general.

Appendix A. The case with no potential. Consider the nonlinear Schrödinger equation without potential:

$$
i \partial_{t} v+\frac{1}{2} \Delta v=\lambda|v|^{2 \sigma} v ; \quad v_{\mid t=0}=v_{0},
$$


with energy-subcritical or energy-critical nonlinearity, $\sigma \leqslant 2 /(d-2)$ if $d \geqslant 3$.

Lemma A.1. Let $\sigma \in \mathbf{N}$ with $\sigma \geqslant 2$ if $d=1$, and $\sigma \leqslant 2 /(d-2)$ if $d \geqslant 3$. Let

$$
\left(p_{1}, q_{1}\right)=\left(2 \sigma+2, \frac{2 d(\sigma+1)}{d-2+d \sigma}\right) .
$$

Assume that (A.1) possesses a global solution $v \in L^{p_{1}}\left(\mathbf{R} ; W^{1, q_{1}}\left(\mathbf{R}^{d}\right)\right)$. Let $k \in \mathbf{N}$. If $v_{0} \in H^{k}\left(\mathbf{R}^{d}\right)$, then $v \in L^{\infty}\left(\mathbf{R} ; H^{k}\left(\mathbf{R}^{d}\right)\right)$, and more generally, $v \in L^{p_{0}}\left(\mathbf{R} ; W^{k, q_{0}}\left(\mathbf{R}^{d}\right)\right)$ for all admissible pairs $\left(p_{0}, q_{0}\right)$.

Remark A.2. The assumption $\sigma \in \mathbf{N}$ is made only to simplify the presentation. The proof could be adapted to the case where the map $z \mapsto|z|^{2 \sigma} z$ is $C^{k}$.

REMARK A.3. The main assumption of the lemma states essentially that asymptotic completeness holds in a suitable space. We could even assume that the nonlinearity is $(2 \sigma+1)$-homogeneous, and not necessarily gauge invariant. However, scattering is known with no size assumption on $v_{0}$ in the defocusing gauge invariant case (see below), hence our choice. Note that in the case $d=1$, an algebraic control of the growth of Sobolev norms is known, regardless of gauge invariance [32].

Proof. We remark that the pair $\left(p_{1}, q_{1}\right)$ is admissible, and

$$
\frac{1}{p_{1}^{\prime}}=\frac{2 \sigma+1}{p_{1}} ; \quad \frac{1}{q_{1}^{\prime}}=\frac{1}{q_{1}}+\frac{2 \sigma}{d \sigma(\sigma+1)} .
$$

We prove the lemma by induction on $k$. We first prove

$$
v \in L^{p_{1}}\left(\mathbf{R} ; W^{k, q_{1}}\right) \cap L^{\infty}\left(\mathbf{R} ; H^{k}\right) .
$$

We start with $k=1$ : applying $\nabla$ to (A.1), Strichartz estimates on $I=\left[t_{0}, t\right]$ yield

$$
\begin{aligned}
\|\nabla v\|_{L^{\infty}\left(I ; L^{2}\right) \cap L^{p_{1}\left(I ; L^{q_{1}}\right)}} & \leqslant C\left(\left\|\nabla v\left(t_{0}\right)\right\|_{L^{2}}+\left\||v|^{2 \sigma} \nabla v\right\|_{L^{p_{1}^{\prime}\left(I ; L^{q_{1}^{\prime}}\right)}}\right) \\
& \leqslant C\left(\left\|v\left(t_{0}\right)\right\|_{L^{2}}+\|v\|_{L^{p_{1}\left(I ; L^{d \sigma(\sigma+1)}\right)}}^{2 \sigma}\|\nabla v\|_{L^{p_{1}\left(I ; L^{q_{1}}\right)}}\right),
\end{aligned}
$$

where we have used Hölder's inequality. Notice the embedding

$$
W^{1, q_{1}}\left(\mathbf{R}^{d}\right) \subset L^{d \sigma(\sigma+1)}\left(\mathbf{R}^{d}\right) .
$$

In view of the assumption of the lemma, this implies $v \in L^{p_{1}}\left(\mathbf{R} ; L^{d \sigma(\sigma+1)}\right)$. Therefore, we can split $\mathbf{R}$ into finitely many intervals on which $C\|v\|_{L^{p_{1}\left(I ; L^{d \sigma(\sigma+1)}\right)}}^{2 \sigma} \leqslant 1 / 2$. On each such interval $I$, we have

$$
\|\nabla v\|_{L^{\infty}\left(I ; L^{2}\right) \cap L^{p_{1}}\left(I ; L^{q_{1}}\right)} \leqslant 2 C\left\|\nabla v\left(t_{0}\right)\right\|_{L^{2}} .
$$

The conclusion follows in the case $k=1$.

Assume now that the result is known for $k \geqslant 1$, and that the nonlinearity is $C^{k+1}$. Differentiating (A.1) $k+1$ times with respect to space variable, we find, for $|\alpha|=k+1$,

$$
\left(i \partial_{t}+\frac{1}{2} \Delta\right) \partial^{\alpha} v=N_{1}(v)+N_{2}(v)
$$

with the pointwise controls

$$
\left|N_{1}(v)\right| \lesssim|v|^{2 \sigma}\left|\partial^{\alpha} v\right| \quad ; \quad\left|N_{2}(v)\right| \lesssim \sum_{\left|\alpha_{j}\right| \leqslant k}\left|\partial^{\alpha_{1}} v\right| \ldots\left|\partial^{\alpha_{2 \sigma+1}} v\right| .
$$


Strichartz estimates on the time interval $I=\left[t_{0}, t\right]$ yield

$$
\begin{aligned}
&\left\|\partial^{\alpha} v\right\|_{L^{\infty}\left(I ; L^{2}\right) \cap L^{p_{1}}\left(I ; L^{q_{1}}\right)} \lesssim\left\|\partial^{\alpha} v\left(t_{0}\right)\right\|_{L^{2}}+\sum_{j=1,2}\left\|N_{j}(v)\right\|_{L^{p_{1}^{\prime}}\left(I ; L^{q_{1}^{\prime}}\right)} \\
& \lesssim\left\|\partial^{\alpha} v\left(t_{0}\right)\right\|_{L^{2}}+\|v\|_{L^{p_{1}}\left(I ; L^{q_{2}}\right)}^{2 \sigma}\left\|\partial^{\alpha} v\right\|_{L^{p_{1}}\left(I ; L^{q_{2}}\right)} \\
&+\sum_{J}\left\|\partial^{\alpha_{1}} v\right\|_{L^{p_{1}}\left(I ; L^{q_{2}}\right)} \ldots\left\|\partial^{\alpha_{2 \sigma}} v\right\|_{L^{p_{1}\left(I ; L^{q_{2}}\right)}}\left\|\partial^{\alpha_{2 \sigma+1}} v\right\|_{L^{p_{1}\left(I ; L^{q_{1}}\right)}},
\end{aligned}
$$

where we have denoted $q_{2}=d \sigma(\sigma+1)$, and we have used the ordering

$$
J=\left\{\left|\alpha_{1}\right|, \ldots,\left|\alpha_{2 \sigma-1}\right| \leqslant k-1 ;\left|\alpha_{2 \sigma}\right|,\left|\alpha_{2 \sigma+1}\right| \leqslant k ; \quad \sum \alpha_{j}=\alpha\right\} .
$$

Proceeding as in the case $k=1$, we consider finitely many time intervals on which

$$
\begin{aligned}
\left\|\partial^{\alpha} v\right\|_{L^{\infty}\left(I ; L^{2}\right) \cap L^{p_{1}}\left(I ; L^{q_{1}}\right)} \lesssim\left\|\partial^{\alpha} v\left(t_{0}\right)\right\|_{L^{2}} \\
\quad+\sum_{J}\left\|\partial^{\alpha_{1}} v\right\|_{L^{p_{1}}\left(I ; L^{q_{2}}\right)} \ldots\left\|\partial^{\alpha_{2 \sigma}} v\right\|_{L^{p_{1}\left(I ; L^{q_{2}}\right)}}\left\|\partial^{\alpha_{2 \sigma+1}} v\right\|_{L^{p_{1}\left(I ; L^{q_{1}}\right)}} .
\end{aligned}
$$

We use the embedding $W^{1, q_{1}} \subset L^{q_{2}}$ and the induction assumption: when $\alpha_{2 \sigma}=k$, we proceed like for the term $N_{1}$ (when summing over all $\alpha$ 's such that $|\alpha|=k+1$ ), and in all the other cases, we deal with a controllable source term. This yields the lemma for the pair $\left(p_{0}, q_{0}\right)=(\infty, 2)$. The estimate for general admissible pairs follows by using Strichartz estimates again.

Proposition A.4. Let $\lambda>0$, and let $\sigma \geqslant 2 / d$ be an integer, with $\sigma \leqslant 2 /(d-2)$ if $d \geqslant 3$. Suppose $v_{0} \in \Sigma$. Let $k \in \mathbf{N}, k \geqslant 1$.

(i) If $v_{0} \in H^{k}\left(\mathbf{R}^{d}\right)$, then $v \in L^{p_{0}}\left(\mathbf{R} ; W^{k, q_{0}}\left(\mathbf{R}^{d}\right)\right)$ for all admissible pairs $\left(p_{0}, q_{0}\right)$.

(ii) If in addition $x \mapsto|x|^{k} v_{0} \in L^{2}\left(\mathbf{R}^{d}\right)$, then $|x|^{k} v \in C\left(\mathbf{R} ; L^{2}\left(\mathbf{R}^{d}\right)\right)$ and for all admissible pairs $\left(p_{0}, q_{0}\right)$,

$$
\forall \alpha \in \mathbf{N}^{d},|\alpha| \leqslant k, \quad\left\|x^{\alpha} v\right\|_{L^{p_{0}\left([0, t] ; L^{q_{0}}\right)}} \lesssim\langle t\rangle^{|\alpha|} .
$$

Remark A.5. In the case $\sigma>2 / d,(i)$ remains valid without assuming $v_{0} \in \Sigma$. The point to notice is that one can apply Lemma A.1 as in the proof below, since the assumptions of the lemma are known to be satisfied thanks to Morawetz estimates, which yield asymptotic completeness in $H^{1}$. In the case $\sigma=2 / d$, this aspect is still an open question.

Proof. Under our assumptions on $\lambda$ and $\sigma$, we know that there exists a unique global solution $v \in C(\mathbf{R} ; \Sigma)$ to (A.1), with $v \in L^{\infty}\left(\mathbf{R} ; H^{1}\right)$. The pseudo-conformal conservation law yields

$$
\frac{d}{d t}\left(\frac{1}{2}\|J(t) v\|_{L^{2}}^{2}+\frac{\lambda t^{2}}{\sigma+1}\|v\|_{L^{2 \sigma+2}}^{2 \sigma+2}\right)=\frac{t \lambda}{\sigma+1}(2-d \sigma)\|v\|_{L^{2 \sigma+2}}^{2 \sigma+2}
$$

where $J(t)=x+i t \nabla$. The right hand side is non-positive for $t \geqslant 0$ : this yields an $a$ priori bound for $J(t) v$ in $L^{\infty}\left(\mathbf{R} ; L^{2}\right)$. Since

$$
J(t)=i t e^{i \frac{|x|^{2}}{2 t}} \nabla\left(e^{-i \frac{|x|^{2}}{2 t}}\right)
$$


the Gagliardo-Nirenberg inequality yields

$$
\|v\|_{L^{\rho}} \lesssim \frac{1}{|t|^{\delta}}\|v\|_{L^{2}}^{1-\delta}\|J(t) v\|_{L^{2}}^{\delta}, \quad \text { where } \delta=d\left(\frac{1}{2}-\frac{1}{\rho}\right), \text { and } 2 \leqslant \rho \leqslant \frac{2 d}{d-2} .
$$

We infer $v \in L^{p_{1}}\left(\mathbf{R} ; L^{q_{1}}\right)$. Resume the value

$$
\theta=\frac{4 \sigma(\sigma+1)}{2-(d-2) \sigma} \quad\left(\theta=\infty \text { if } \sigma=\frac{2}{d-2}\right) .
$$

In view of the identities

$$
(p, q)=\left(\frac{4 \sigma+4}{d \sigma}, 2 \sigma+2\right) ; \quad \frac{1}{p^{\prime}}=\frac{1}{p}+\frac{2 \sigma}{\theta} ; \quad \frac{1}{q^{\prime}}=\frac{2 \sigma+1}{q},
$$

Strichartz estimates yield

$$
\|\nabla v\|_{L^{p}\left(I ; L^{q}\right) \cap L^{p_{1}\left(I ; L^{q_{1}}\right)}} \lesssim 1+\|v\|_{L^{\theta}\left(I ; L^{q}\right)}^{2 \sigma}\|\nabla v\|_{L^{p}\left(I ; L^{q}\right)} .
$$

We note that $v \in L^{\theta}\left(\mathbf{R} ; L^{q}\right)$ (and $\|v(t)\|_{L^{q}} \rightarrow 0$ uniformly as $t \rightarrow \infty$ ): splitting $\mathbf{R}$ into finitely many intervals, we infer that $\nabla v \in L^{p_{1}}\left(\mathbf{R} ; L^{q_{1}}\right)$ : the first point of the proposition then follows from Lemma A.1.

The second point of the proposition is obtained by mimicking the proof of Lemma A.1: instead of considering $\nabla$ and its powers, consider $J=x+i t \nabla$ and its powers. In view of (A.2), we can follow the same computations since the nonlinearity we consider is gauge invariant: $|J|^{k} v \in L^{\infty}\left(\mathbf{R} ; L^{2}\right)$. The algebraic growth of the momenta then stems from triangle inequality.

Acknowledgment. The author wishes to thank Clotilde Fermanian for fruitful discussions on this subject, and Lysianne Hari for a careful reading of the manuscript.

\section{REFERENCES}

[1] M.J. Ablowitz and P.A. Clarkson, Solitons, Nonlinear Evolution Equations and Inverse Scattering, London Mathematical Society Lecture Note Series, Cambridge University Press, Cambridge, 149, 1991.

[2] P. Bégout, Convergence to scattering states in the nonlinear Schrödinger equation, Commun. Contemp. Math., 3(3), 403-418, 2001.

[3] J. Bourgain, Global Solutions of Nonlinear Schrödinger Equations, American Mathematical Society Colloquium Publications, American Mathematical Society, Providence, RI, 46, 1999.

[4] A. Bouzouina and D. Robert, Uniform semiclassical estimates for the propagation of quantum observables, Duke Math. J., 111(2), 223-252, 2002.

[5] R. Carles, Critical nonlinear Schrödinger equations with and without harmonic potential, Math. Models Methods Appl. Sci., 12(10), 1513-1523, 2002.

[6] R. Carles, Nonlinear Schrödinger equations with repulsive harmonic potential and applications, SIAM J. Math. Anal., 35(4), 823-843, 2003.

[7] R. Carles, Global existence results for nonlinear Schrödinger equations with quadratic potentials, Discrete Contin. Dyn. Syst., 13(2), 385-398, 2005.

[8] R. Carles, Linear vs. nonlinear effects for nonlinear Schrödinger equations with potential, Commun. Contemp. Math., 7(4), 483-508, 2005.

[9] R. Carles, On the Cauchy problem in Sobolev spaces for nonlinear Schrödinger equations with potential, Portugal. Math. (N. S.), 65(2), 191-209, 2008.

[10] R. Carles, Rotating points for the conformal NLS scattering operator, Dyn. Partial Differ. Equ., $6(1), 35-51,2009$. 
[11] R. Carles and C. Fermanian-Kammerer, Nonlinear coherent states and ehrenfest time for Schrödinger equations, Commun. Math. Phys., 301(2), 443-472, 2011.

[12] Y. Castin and R. Dum, Bose-Einstein condensates in time dependent traps, Phys. Rev. Lett., $77(27), 5315,1996$.

[13] T. Cazenave, Semilinear Schrödinger Equations, Courant Lecture Notes in Mathematics, New York University Courant Institute of Mathematical Sciences, New York, 10, 2003.

[14] T. Cazenave and F. Weissler, Rapidly decaying solutions of the nonlinear Schrödinger equation, Commun. Math. Phys., 147, 75-100, 1992.

[15] M. Combescure and D. Robert, Semiclassical spreading of quantum wave packets and applications near unstable fixed points of the classical flow, Asymptot. Anal., 14(4), 377-404, 1997.

[16] R. Cordero-Soto, R.M. Lopez, E. Suazo, and S.K. Suslov, Propagator of a charged particle with a spin in uniform magnetic and perpendicular electric fields, Lett. Math. Phys., 84(2-3), 159-178, 2008.

[17] W. Craig, T. Kappeler, and W. Strauss, Microlocal dispersive smoothing for the Schrödinger equation, Commun. Pure Appl. Math., 48(8), 769-860, 1995.

[18] H.L. Cycon, R.G. Froese, W. Kirsch, and B. Simon, Schrödinger Operators with Application to Quantum Mechanics and Global Geometry, study ed., Texts and Monographs in Physics, Springer-Verlag, Berlin, 1987.

[19] R.P. Feynman and A.R. Hibbs, Quantum Mechanics and Path Integrals (International Series in Pure and Applied Physics), McGraw-Hill Publishing Company, Ltd., 1965.

[20] D. Fujiwara, A construction of the fundamental solution for the Schrödinger equation, J. Analyse Math., 35, 41-96, 1979.

[21] D. Fujiwara, Remarks on the convergence of the Feynman path integrals, Duke Math. J., 47(3), 559-600, 1980.

[22] J.J. García-Ripoll, V.M. Pérez-García, and V. Vekslerchik, Construction of exact solutions by spatial translations in inhomogeneous Schrödinger equations, Phys. Rev. E, 64, 056602, 2001.

[23] L. Hörmander, Symplectic classification of quadratic forms, and general Mehler formulas, Math. Z., 219(3), 413-449, 1995.

[24] L. Kapitanski, I. Rodnianski, and K. Yajima, On the fundamental solution of a perturbed harmonic oscillator, Topol. Methods Nonlinear Anal., 9, 1, 77-106, 1997.

[25] O. Kavian and F. Weissler, Self-similar solutions of the pseudo-conformally invariant nonlinear Schrödinger equation, Michigan Math. J., 41(1), 151-173, 1994.

[26] M. Keel and T. Tao, Endpoint Strichartz estimates, Amer. J. Math., 120(5), 955-980, 1998.

[27] U Al Khawaja, Soliton localization in Bose-Einstein condensates with time-dependent harmonic potential and scattering length, J. Phys. A: Math. Theor., 42, 265206, 2009.

[28] V. Ramesh Kumar, R. Radha, and Prasanta K. Panigrahi, Dynamics of Bose-Einstein condensates in a time-dependent trap, Phys. Rev. A, 77, 023611, 2008.

[29] P. Raphaël, On the blow up phenomenon for the $L^{2}$ critical non linear Schrödinger equation, Lectures on nonlinear dispersive equations, GAKUTO Internat. Ser. Math. Sci. Appl., Gakkōtosho, Tokyo, 27, 9-61, 2006.

[30] A.V. Rybin, G.G. Varzugin, M. Lindberg, J. Timonen, and R.K. Bullough, Similarity solutions and collapse in the attractive Gross-Pitaevskii equation, Phys. Rev. E(3), part A, 62(5), 6224-6228, 2000.

[31] V.N. Serkin, Akira Hasegawa, and T.L. Belyaeva, Nonautonomous solitons in external potentials, Phys. Rev. Lett., 98(7), 074102, 2007.

[32] G. Staffilani, On the growth of high Sobolev norms of solutions for KdV and Schrödinger equations, Duke Math. J., 86(1), 109-142, 1997.

[33] C. Sulem and P.L. Sulem, The Nonlinear Schrödinger Equation, Self-focusing and Wave Collapse, Springer-Verlag, New York, 1999.

[34] T. Tao, Nonlinear Dispersive Equations, CBMS Regional Conference Series in Mathematics, Published for the Conference Board of the Mathematical Sciences, Washington, DC, Local and global analysis., 106, 2006.

[35] T. Tao, A pseudoconformal compactification of the nonlinear Schrödinger equation and applications, New York J. Math., 15, 265-282, 2009.

[36] Y. Tsutsumi, Scattering problem for nonlinear Schrödinger equations, Ann. Inst. H. Poincaré Phys. Théor., 43(3), 321-347, 1985.

[37] Y. Tsutsumi, $L^{2}$-solutions for nonlinear Schrödinger equations and nonlinear groups, Funkcial. Ekvac., 30(1), 115-125, 1987.

[38] Baoxiang Wang, Nonlinear scattering for a class of wave equations in $H^{s}$, J. Math. Anal. Appl., 296, 74-96, 2004. 
[39] K. Yajima, Smoothness and non-smoothness of the fundamental solution of time dependent Schrödinger equations, Commun. Math. Phys., 181(3), 605-629, 1996.

[40] S. Zelditch, Reconstruction of singularities for solutions of Schrödinger's equation, Commun. Math. Phys., 90(1), 1-26, 1983. 